
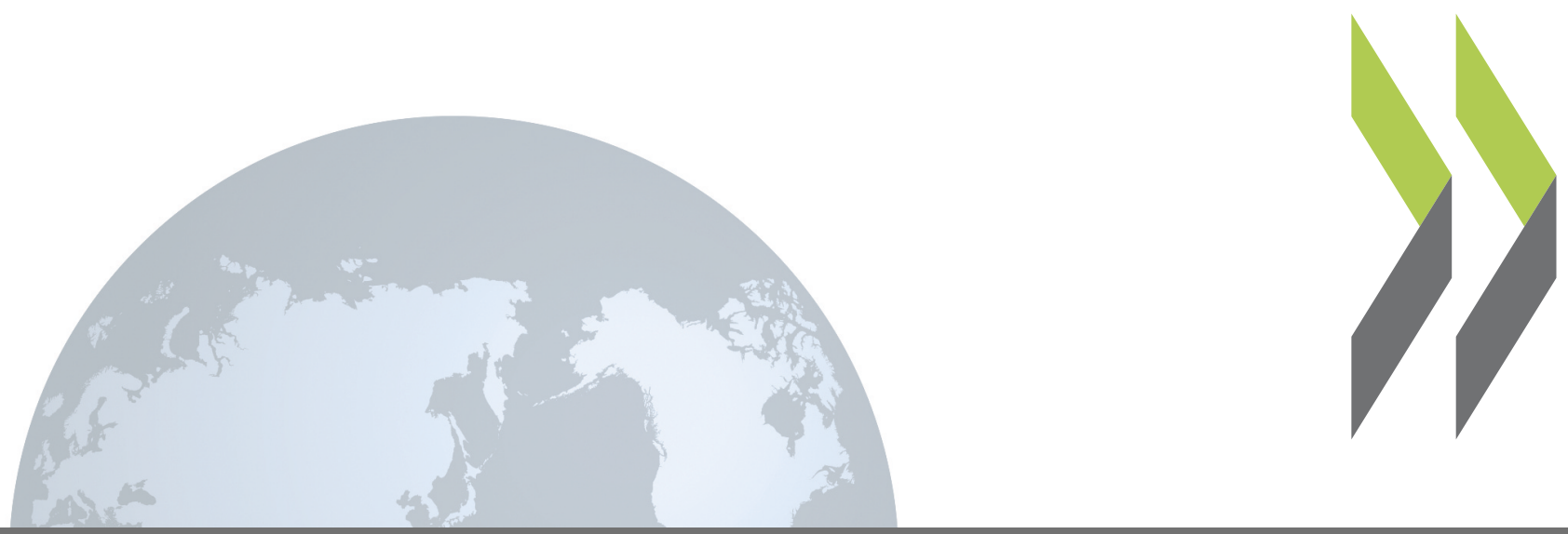

Documents SIGMA No. 35

\title{
Coordination des centres du gouvernement :
} Fonctions et organisation du bureau du gouvernement Analyse comparative des pays membres de l'OCDE, des PECO et des pays des Balkans occidentaux OCDE 
SIGMA

Soutien à l'amélioration des institutions publiques et des systèmes de gestion Initiative conjointe de l'OCDE et l'UE, principalement financée par l'UE

\section{COORDINATION DES CENTRES DU GOUVERNEMENT : FONCTIONS ET ORGANISATION DU BUREAU DU GOUVERNEMENT ANALYSE COMPARATIVE DES PAYS MEMBRES DE L'OCDE, DES PECO ET DES PAYS DES BALKANS OCCIDENTAUX}

Par Michal Ben-Gera

DOCUMENT SigMA N ${ }^{\circ} 35$

Mars 2004 


\section{LE PROGRAMME SIGMA}

Le Programme Sigma - Soutien à l'amélioration des institutions publiques et des systèmes de gestion — est une initiative conjointe de l'Organisation de coopération et de développement économiques (OCDE) et de l'Union européenne, financée principalement par l'UE.

Sigma soutient les pays partenaires dans leurs efforts pour améliorer leurs institutions publiques et leur système de gestion en :

- évaluant les progrès de la réforme et en définissant des priorités par rapport aux références que constituent les bonnes pratiques européennes et la législation existante au sein de l'UE (l'acquis communautaire) ;

- $\quad$ assistant les décideurs et les administrations dans la mise en place de l'organisation et des procédures d'adaptation aux normes et bonnes pratiques européennes ;

- $\quad$ aidant les donateurs intra et extra-européens à concevoir des projets, à instaurer les conditions préalables à leur réalisation et en les assistant dans leur mise en œuvre.

Le Programme Sigma travaille en partenariat avec les gouvernements des pays suivants :

- La plupart des pays candidats à l'UE - Bulgarie, République tchèque, Estonie, Hongrie, Lettonie, Lituanie, Pologne, Roumanie, Slovaquie, Slovénie et Turquie.

- Pays des Balkans occidentaux - Albanie, Bosnie-Herzégovine, Croatie, ancienne République yougoslave de Macédoine et la Serbie-et-Monténégro/Monténégro, Serbie et Kosovo.

- $\quad$ La Russie (sous le financement de l'OCDE).

Le programme Sigma a fixé ses priorités afin de soutenir les initiatives de réforme des pays partenaires dans les domaines suivants :

- Conception et application des programmes de réforme

- $\quad$ Structures juridiques, fonction publique et justice

- $\quad$ Audit externe et contrôle financier

- Gestion de la dépense publique

- Capacités d'élaboration des politiques et coordination, y compris la gestion de la réglementation

- $\quad$ Marchés publics

Pour plus d'informations sur le programme Sigma, consultez notre site Internet :

http://www.sigmaweb.org

(C) OCDE 2004

Ceci est une traduction à partir du titre original : Co-ordination at the Centre of Government: The Functions and

Organisation of the Government Office [GOV/SIGMA(2004)2].

Toute demande d'autorisation, de reproduction ou de traduction de cette publication à usage commercial ou noncommercial devra être envoyée à rights@oecd.org. 


\begin{abstract}
AVANT-PROPOS
La plupart des pays des Balkans occidentaux n'ont commencé que très récemment à adapter leurs institutions et leurs cadres juridiques aux nécessités et aux conditions d'une économie de marché occidentale. Pourtant, la complexité des exigences prescrites par le processus de stabilisation et d'association et, plus encore, par l'adhésion à l'UE, requiert des moyens particulièrement développés en coordination, en suivi et en évaluation des politiques. Dans pratiquement tous les pays des Balkans occidentaux ainsi que dans plusieurs nouveaux États membres, les moyens dont disposent les gouvernements et les administrations pour fixer les priorités de politique, développer des options de politique, analyser les politiques, contrôler l'application des politiques et évaluer les politiques demeurent faibles.

Dans les pays des Balkans occidentaux, les ministères et le secrétariat du gouvernement (Bureau du Gouvernement) ne semblent pas pleinement comprendre le rôle qu'ils sont censés jouer dans le processus d'élaboration, de coordination, de suivi et d'évaluation des politiques. Leurs tâches sont essentiellement perçues comme administratives, c.-à-d. exécuter les ordres du gouvernement ou du ministre. Seule exception à cette règle, le ministère ou la direction en charge de l'Intégration européenne (EI), qui néanmoins ne peut se substituer à un bureau du gouvernement en exercice et qui, si telle était son intention, pourrait même générer un déséquilibre parmi les ministères. Dans tous les cas, alors que les centres du gouvernement et les ministères de tutelle manquent cruellement de moyens professionnels en matière de politique, le fait que de tels moyens existent ou non dans un ministère d'IE ne garantit en rien que les priorités choisies soient les plus appropriées ni que les solutions poursuivies soient les plus adaptées au pays. Sans ces deux conditions préalables, toute application peut s'avérer à son tour trop coûteuse et inefficace.
\end{abstract}

Dans les pays membres de l'OCDE ainsi que dans toutes les démocraties occidentales développées, le bureau du gouvernement notamment est un acteur majeur dans la coordination et l'évaluation des politiques. Il est en réalité le garant d'une politique gouvernementale de cohésion en phase avec les priorités gouvernementales fixées.

La présente publication a été dans un premier temps préparée pour servir de référence à un atelier Sigma destiné aux pays des Balkans occidentaux sur le rôle des bureaux des gouvernements. Sur demande des participants à cet atelier, la base des informations ayant servi à la préparation de ce document a été élargie et mise à jour. Le contenu de la présente publication se fonde donc sur les précédents travaux réalisés par la Direction de la gouvernance publique et du développement territorial de l'OCDE (GOV) et Sigma. Les deux organisations envisagent de développer une base de données commune GOV/Sigma sur les structures et les tâches des centres du gouvernement $(\mathrm{CdG})$ dans le but de fournir des éléments de comparaison pour les réseaux de l'OCDE et de Sigma.

Cette publication s'adresse principalement aux bureaux/secrétariats gouvernementaux des pays des Balkans occidentaux, mais peut également s'avérer utile pour d'autres pays en pleine transition visant à améliorer leurs capacités en matière de coordination des politiques. 


\section{NOTE DE SYNTHÈSE}

Un bureau de gouvernement pleinement opérationnel agit en qualité de coordonnateur du système décisionnel et, en tant que tel, s'inscrit comme une composante essentielle des capacités d'un gouvernement à définir et à poursuivre ses objectifs collectifs.

Le «bureau du gouvernement» est un terme générique qui se rapporte aux institutions des centres du gouvernement chargées de soutenir le Premier ministre et de servir le Conseil des ministres en leur qualité d'organes collectifs de prise de décisions. Le présent document s'attache à décrire et à analyser les fonctions et l'organisation des bureaux de gouvernement, en comparant les pays membres de l'OCDE, les pays de l'Europe centrale et orientale (PECO) et les pays des Balkans occidentaux (ancienne Yougoslavie et Albanie). Cette analyse se fonde sur les informations recueillies par Sigma et la GOV (PUMA) ${ }^{1}$ depuis le milieu des années 90, complétées par les résultats d'un questionnaire écrit administré par Sigma et la GOV en 2003.

\section{Fonctions :}

La complexité des gouvernements modernes - aussi bien en termes de thèmes traités que d'organisation rend nécessaire l'instauration d'un point de référence pour la coordination. Une responsabilité qui incombe principalement au Bureau du Gouvernement. Organe ayant pour première vocation d'assurer la coordination, le Bureau du Gouvernement a pour tâche principale de s'assurer que les diverses activités de chaque ministère et agence sont exécutées de manière efficace et cohérente. Au sein de ce cadre, le présent document s'emploie à analyser et à comparer les fonctions du Bureau du Gouvernement dans les divers pays mentionnés suivant huit axes de coordination :

- Coordination de la préparation des sessions du Conseil des ministres, y compris la préparation de l'ordre du jour et la diffusion des documents aux participants ;

- Coordination des activités pour assurer la conformité juridique, y compris la conformité des projets de lois avec la Constitution et le corps législatif existant ;

- Coordination de la préparation et de l'approbation des priorités stratégiques et du programme de travail du gouvernement, et de leur dotation dans le budget ;

- Coordination du contenu des propositions de politique soumises au Conseil des ministres pour décision, y compris la définition du processus de préparation des politiques par les ministères, la coordination interministérielle et l'ajustement des propositions entre elles et en fonction des priorités du gouvernement ;

- Coordination des activités de communication du gouvernement pour assurer la cohérence du message gouvernemental, ainsi que la synchronisation et le contenu efficaces des messages ministériels ;

- Coordination du suivi de la performance gouvernementale pour s'assurer que l'exécution collective du gouvernement est efficace et tient ses promesses envers le public ;

- Coordination des échanges entre le gouvernement et les autres acteurs de l'état (Président, Parlement) ; et

- Coordination des priorités stratégiques horizontales spécifiques, telles que la réforme de l'administration publique, l'intégration européenne, ou les rapports intergouvernementaux dans les fédérations.

Il ressort de cette analyse que la majorité des Bureaux de gouvernement assurent la plupart de ces tâches. Au cours de ces dix dernières années, le fonctionnement des Bureaux de gouvernement des PECO n'a cessé de se rapprocher de celui des Bureaux de gouvernement des pays de l'OCDE. Généralement, les Bureaux de gouvernement dans les pays des Balkans occidentaux souffrent de retards dans l'exécution de certaines fonctions, notamment celles liées à la planification et à la coordination des politiques.

1 Le Service de la gestion publique de l'OCDE (PUMA) est devenu la Direction de la gouvernance publique et du développement territorial (GOV) en septembre 2002. 


\section{Structure :}

Si la structure des Bureaux de gouvernement varie considérablement, ces différences n'en dissimulent pas moins des similarités fondamentales. S'il n'existe pas de Bureau du Gouvernement générique, il est très facile d'en esquisser un modèle général dans lequel presque chaque pays pourrait trouver une similitude significative avec sa propre structure. La majorité des Bureaux de gouvernement sont présidés par un Ministre ou un Secrétaire Général (qui est nommé soit par le gouvernement, soit par le Premier ministre). Dans la majorité des cas, le Secrétaire Général (même s'il exerce en tant que fonctionnaire) est sujet à remplacement lorsqu'un nouveau Premier ministre est nommé. La plupart des Bureaux de gouvernement exercent à la fois un soutien politique et logistique directement destiné au Premier ministre et assiste le Conseil des ministres en tant qu'organe collectif. Dans certains cas, on distingue deux organisations individuelles, alors que dans d'autres cas, on n'observe qu'une seule structure qui opère toutefois une distinction interne entre fonctionnaires et nominataires politiques.

Un Bureau de gouvernement s'organise en règle générale autour d'unités combinées (départements, secteurs, bureaux, etc.), chacune étant chargée d'exécuter une ou plusieurs fonctions de coordination. On observe en général les principales unités suivantes :

- $\quad$ Le Cabinet du Premier ministre (CPM) : Il s'agit du cabinet politique qui fournit le soutien logistique et politique spécifiquement destiné au Premier ministre ;

- Les Cabinets des vice-Premiers ministres (s'ils existent) et les cabinets des autres ministres sans portefeuille ;

- L'Unité dédiée à la presse et aux communications : Dans de nombreux cas, cette unité est rattachée au CPM, essentiellement en raison de la nature politique des communications ;

- L'Unité chargée de la préparation technique/logistique des sessions du Conseil des ministres et des réunions des comités ministériels (s'ils existent);

- L'Unité ou les unités chargées d'assurer la coordination des politiques et de conseiller le Conseil des ministres, le Premier ministre et les Présidents des comités : dans de nombreux cas, on observe entre trois et cinq unités de ce type, définies en fonction de thèmes sectoriels (par exemple, politique économique, politique sociale, défense, politique extérieure) ;

- $\quad$ L'Unité de planification : dans certains cas, il n'existe aucune unité distincte de planification, auquel cas le travail de planification incombe à l'unité ou aux unités chargées de la coordination des politiques ;

- $\quad$ L'Unité juridique ou législative ;

- $\quad$ L'Unité chargée de superviser l'application des décisions gouvernementales : lorsque cette supervision s'avère essentiellement technique, cette fonction peut être remplie par l'unité qui prépare les sessions du Conseil des ministres ;

- L'Unité chargée de gérer les échanges entre le gouvernement et le Parlement et/ou le Bureau du Président ;

- Les Unités spécialisées chargées de gérer les questions horizontales substantielles dévolues au Bureau du gouvernement, comme les Affaires européennes ou les relations intergouvernementales (dans les fédérations); et

- L'Unité chargée de l'administration interne du Bureau du gouvernement (personnel, budget, informatique, etc.).

\section{Réforme des bureaux de gouvernement :}

Le présent document arrive à la conclusion qu'il est possible et souvent souhaitable de réformer le Bureau du gouvernement dans les pays en transition. Les principales leçons à tirer de telles réformes sont les suivantes :

- Il y a un besoin d'engagement au plus haut niveau dans la réforme, idéalement de la part du Premier ministre, mais au minimum au niveau du Secrétaire Général.

- Il est préférable de débuter la réforme et de bâtir de nouvelles structures à partir de l'organisation existante que de repartir de zéro ou d'appliquer le modèle d'un autre pays. Il vaut mieux consacrer du temps et des efforts dans une évaluation initiale pour dresser un aperçu de la situation en cours, puis élaborer des idées de réforme sur la base de cette évaluation. 
- L'aide extérieure s'avère souvent utile mais, à moins de relations de travail étroites entre la direction et le personnel du Bureau du gouvernement, cette aide extérieure perd cruellement de sa valeur après l'évaluation initiale.

- $\quad$ Tout changement prend du temps à se mettre en place, étant donné que le processus de mise en œuvre s'avère souvent difficile et implique des décisions du Premier ministre et du gouvernement, des efforts de planification, des changements législatifs/réglementaires, des ressources et de la formation. Ce processus peut prendre un an ou plus avant de commencer à porter ses fruits, et pas moins de deux à trois ans pour achever l'application des changements visés.

- La réforme du Bureau du gouvernement doit se faire en parallèle avec le renforcement des moyens en matière de politique dans les ministères. L'action du Bureau du gouvernement dépend dans une large mesure des documents en provenance des ministères. Si les ministères n'ont pas les moyens d'élaborer des propositions de haute qualité, de se consulter et d'en évaluer les impacts, le Bureau du gouvernement n'est pas en mesure de garantir une prise de décision éclairée.

- Une réforme réussie exige un changement dans la culture de l'organisation. Le Bureau du gouvernement a besoin de fonctionnaires capables d'aller au delà de l'application des règles et de fournir des jugements éclairés sur les conséquences des options soumises au Conseil des ministres pour décision.

Le message de la présente publication est qu'il est possible et grandement souhaitable de réformer les Bureaux de gouvernement des pays en transition en renforçant leurs moyens en termes de politique et de planification, de sorte que les gouvernements parviennent à gérer les multiples tâches résultant de la transition. 


\section{TABLE DES MATIÈRES}

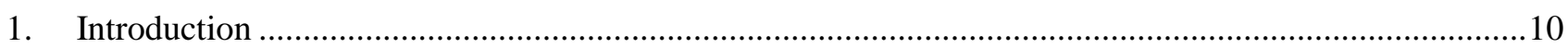

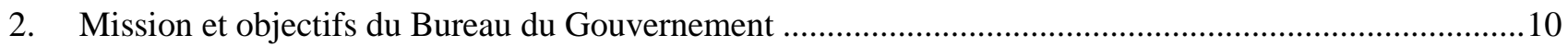

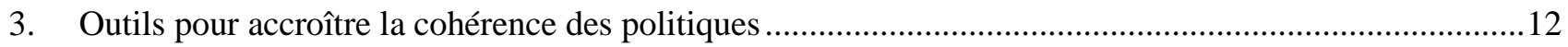

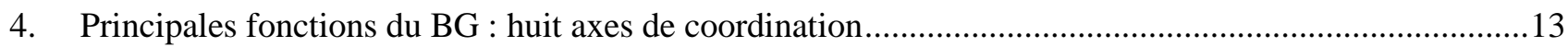

4.1 Coordination de la préparation des sessions du gouvernement .....................................................14

4.1.1 Délais de soumission et de diffusion des documents pour les sessions ...................................14

4.1.2 L'ordre du jour des sessions du CdM.............................................................................. 18

4.1.3 Examen des documents pour s'assurer de l'exhaustivité des dossiers et de l'observation des

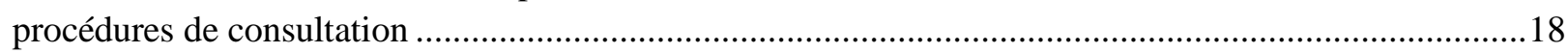

4.2 Coordination de la conformité juridique ...................................................................................21

4.3 Coordination de la préparation du programme gouvernemental (priorités stratégiques, programmes

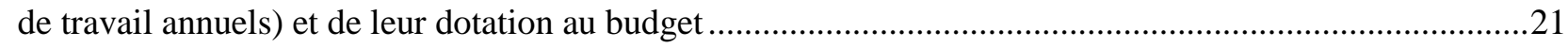

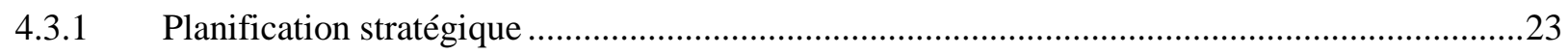

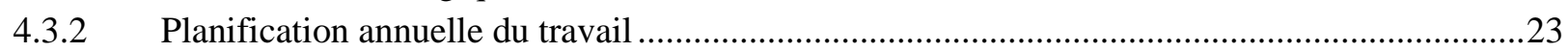

4.4 Coordination du contenu politique des propositions pour le $\mathrm{CdM}$ (préparation dans les ministères,

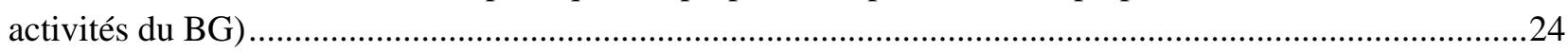

4.4.1 Préparation des documents dans les ministères : rôle du BG dans l'établissement de procédures

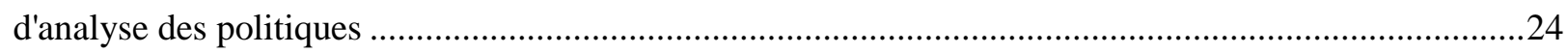

4.4.2 Examen des politiques au sein du BG : coordination et résolution des conflits ......................28

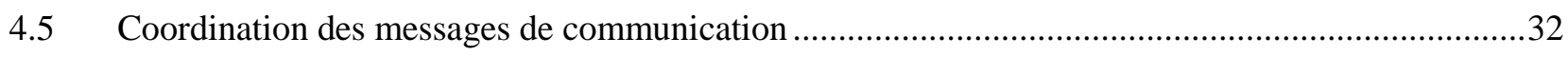

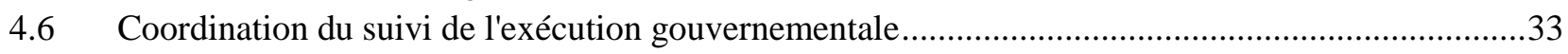

4.7 Coordination des échanges avec les autres instances de l'État (Président, Parlement).....................33

4.8 Coordination des priorités stratégiques horizontales spécifiques (IE, RAP) ......................................34

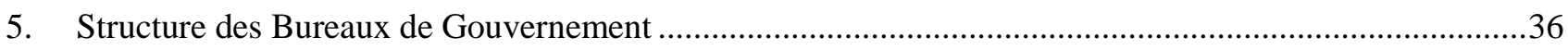

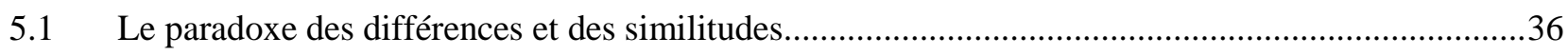

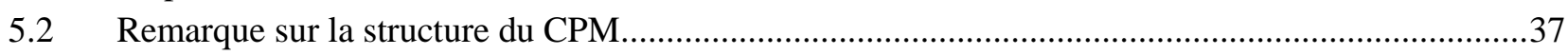

6. Conclusion : Vers un renforcement du Bureau du Gouvernement ...............................................................39

\section{Tableaux}

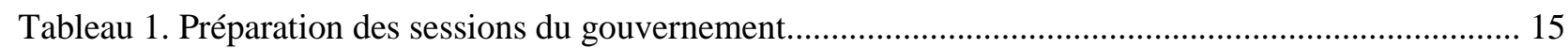

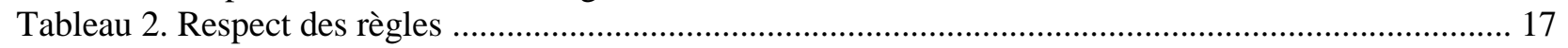

Tableau 3. Motifs du retour des questions aux ministères pour un travail complémentaire....................20

Tableau 4. Capacité de planification stratégique et du travail pour le CdM .............................................. 22

Tableau 5. Réunions de coordination préalables à la réunion du CdM ..................................................... 30

Tableau 6. Conseils du Bureau du Gouvernement sur les politiques ......................................................... 31

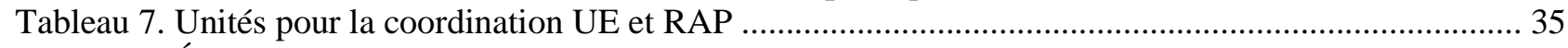

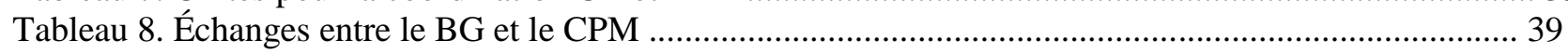




\section{NOTE SUR LA TERMINOLOGIE ET LES ABRÉVIATIONS UTILISÉES DANS CE DOCUMENT}

Gouvernement/Conseil des Ministres (CdM) : Ces termes se substituent l'un à l'autre dans ce document. Ils désignent collectivement les membres du gouvernement. Dans certains pays, le terme de Conseil des Ministres renvoie aux réunions, habituellement hebdomadaires, du gouvernement. Par souci de clarté, les réunions seront désignées ici de manière spécifique, par exemple, la session gouvernementale, la session du CdM.

Bureau du Gouvernement (BG) : Ce terme générique employé dans tout le document renvoie à l'organe administratif rattaché au chef du gouvernement (généralement le Premier ministre) et au Gouvernement/Conseil des Ministres. Le véritable terme employé diffère d'un pays à l'autre, on parle par exemple de Secrétariat général, de Bureau du Gouvernement, de Secrétariat du Gouvernement, de Chancellerie ou de Bureau de Cabinet, etc. Veuillez noter que ce terme est utilisé ici même lorsque l'organe intégral servant le gouvernement est désigné sous le nom de Cabinet du Premier ministre (par exemple, en Pologne, en Australie, au Danemark, en Serbie-Monténégro / Kosovo et aux Pays-Bas).

Le Cabinet du Premier ministre ou le Cabinet (CPM) : Ce terme générique employé dans tout le document renvoie au bureau qui sert spécifiquement le chef du gouvernement, normalement le Premier ministre. Ce bureau est essentiellement politique et fait presque toujours partie du BG, du moins à des fins administratives. Dans certains pays (par exemple, en Bulgarie et en Irlande), ce bureau est relativement modeste et assure essentiellement le soutien logistique, alors que dans d'autres pays (par exemple, en France et au Canada), il est assez conséquent et dispose de moyens significatifs en matière de politique et de conseils politiques.

NOTA : Étant donné la grande diversité dans les structures organisationnelles et dans la terminologie, il est quasiment impossible d'être entièrement sûr de la distinction BG/CPM, sans une étude détaillée du pays concerné. En particulier, les tableaux contenus dans le présent document se basent sur des questionnaires remplis par les pays eux-mêmes, lesquels n'ont certainement pas observé cette distinction de manière aussi constante que dans les propos qui suivent. La présente publication s'efforce d'utiliser ces termes de manière cohérente, comme expliqué ci-dessus. Si l'organe ne sert que le CdM ou à la fois le CdM et le Premier ministre, il est désigné en tant que BG. S'il sert spécifiquement le PM, et si son rôle est essentiellement politique, il sera désigné en tant que CPM. Pour rendre le sujet un peu plus complexe, certains pays désignent leur BG par CPM. Dans les cas où cela se justifie par leurs fonctions, ces organes sont désignés dans le présent document sous le nom de BG.

Politique : Ce terme est particulièrement complexe, en ce sens qu'il n'existe même pas dans toutes les langues. Une politique désigne une approche visant à résoudre un problème ou à améliorer une situation. Elle est souvent englobée dans une loi ou une réglementation, ou même dans un certain nombre de lois et de réglementations, mais peut ne pas l'être. Par exemple, un gouvernement peut annoncer que son principal objectif en matière de politique extérieure est de rejoindre l'OTAN, ou qu'il élabore une politique pour aider les PME via un paquet de mesures fiscales et d'investissement.

Législation, actes juridiques : Il s'agit des outils les plus communs pour faire entrer en vigueur une politique. Un acte juridique englobe une ou des politiques et traite généralement des questions liées à l'application et à l'exécution de cette ou de ces politiques.

Premier ministre : On retrouve dans la terminologie utilisée dans les différents pays le Président du gouvernement, le Président du CdM, le Chancelier et le Taoiseach. Le titre générique de Premier ministre est utilisé dans le présent document pour désigner la personne qui préside le CdM.

Secrétaire d'État : Ce terme est employé de manière générique dans le présent document pour désigner le chef d'un ministère. On observe de grandes différences dans les rôles, les responsabilités, la place occupée dans la hiérarchie et le mode de nomination des secrétaires d'État. Le nom lui-même prête à confusion, d'autant plus que dans certains pays le secrétaire d'état est un titre utilisé pour les ministres juniors.

Secrétaire Général : Ce terme est employé de manière générique dans le présent document pour désigner le chef du Bureau du gouvernement. Là encore, on observe de grandes différences dans les rôles, les responsabilités, le statut et le mode de nomination des Secrétaires Généraux, mais ce terme semble être le plus utilisé et prêter le moins à confusion. 
Comités ministériels : Ce terme désigne les comités officiels des ministres, généralement instaurés dans le but de gérer des secteurs particuliers, comme par exemple les affaires économiques, les affaires sociales, ou des sujets spécifiques, comme l'Intégration européenne, les minorités, l'équité. En règle générale, ces comités sont permanents, du moins pendant toute la durée d'exercice d'un Premier ministre particulier, et sont souvent présidés par les ministres seniors ou par les vice-premiers ministres. Ces comités font souvent office de forums chargés de discuter en profondeur des questions qui les concernent ou de résoudre les désaccords avant de soumettre les questions aux sessions plénières du CdM.

\section{NOTE SUR LES SOURCES D'INFORMATIONS UTILISÉES DANS LE PRÉSENT DOCUMENT}

Les exemples utilisés dans le présent document sont principalement extraits de la publication intitulée «Profils des centres du gouvernement »(PUMA, OCDE, 1996) et des études sur les systèmes décisionnels dans les PECO et les Balkans occidentaux réalisées par Sigma entre 1997 et 2003. Les profils PUMA ont été préparés par les gouvernements participants, et on observe d'importantes différences dans la quantité de détails qu'ils fournissent ainsi que dans la terminologie qu'ils utilisent. (Pour les pays d'Europe centrale et orientale, les principales sources d'analyse sont les rapports et autres évaluations de Sigma diversement préparés par Jacques Fournier, Michal Ben-Gera, Simon James et Tony Verheijen).

Ces informations ont été par la suite revérifiées à la lumière des réponses au questionnaire envoyé aux pays membres de l'OCDE, aux PECO et aux pays des Balkans occidentaux. Ce questionnaire a été rempli par presque tous les gouvernements qui ont ainsi fourni une quantité d'informations sur la structure, les fonctions et le personnel de leur BG et de leur CPM. Toutes les données reportées dans les tableaux proviennent directement des réponses à ce questionnaire. Quoiqu'il en soit, le présent document n'a pas pour vocation de couvrir l'intégralité des informations recueillies dans le questionnaire ni d'analyser de manière poussée la situation d'un pays. En revanche, des exemples viennent illustrer les observations de nature générale.

Les documents analytiques préparés par le PUMA/GOV ont également été consultés, notamment une brève publication sur la cohérence des politiques. Les notes non publiées préparées par Anne Evans et Simon James ont également été une source d'observations utiles sur une première version provisoire du présent document. Benjamin Kumpf a prêté son concours dans la vérification des questionnaires et leur cohérence avec les données précédentes. 


\section{Introduction}

La présente publication a pour objet d'aider les gouvernements des PECO et des pays des Balkans occidentaux à réformer et à renforcer leur Bureau de gouvernement et leur Cabinet du Premier ministre respectifs et ainsi à consolider leur système décisionnel. L'hypothèse sous-tendant la présente démonstration est que les gouvernements peuvent apprendre les uns des autres sur les différentes approches adoptées en matière d'organisation et de dotation en personnel du BG et du CPM. La variété des approches suggère qu'aucun modèle générique ne convient à tous les systèmes, ce qui implique que le principal avantage d'une approche comparative est d'offrir une source d'idées pouvant être prises en considération par ceux qui visent à réformer leur structure. Dans le même temps, il est admis que chaque effort de réforme est unique et qu'il devrait se fonder sur l'analyse en profondeur des conditions du pays en question de manière à adapter les BG et autres CPM locaux aux besoins et aux objectifs d'un système constitutionnel, politique et administratif donné.

Dans les exemples cités dans le présent document, les pays sont regroupés dans l'un des trois groupes suivants : les pays membres de l'OCDE, les pays de l'Europe centrale et orientale (PECO) ou les pays des Balkans occidentaux. Dans la mesure où ces classifications ne s'excluent pas les unes des autres, la décision de classer un pays dans un groupe reste une question de jugement. Par exemple, la Pologne, la Hongrie, la République tchèque et la Slovaquie sont tous des PECO et des pays membres de l'OCDE. Puisque le but de la présente publication est de contribuer à la réforme des $\mathrm{BG}$ dans les nouvelles démocraties, et dans la mesure où ces pays n'ont pas été inclus dans les profils PUMA en 1996, nous avons pris le parti de les traiter comme des PECO dans la présente publication. Dans le présent document, les pays des Balkans occidentaux englobent les pays de l'ex-Yougoslavie (à l'exception de la Slovénie, qui relève du groupe des PECO) ainsi que l'Albanie. Ces groupes incluent certaines entités dont le statut n'est pas encore définitivement déterminé, il est donc question de gouvernements qui existent à ce jour sans préjudice vis-à-vis de tout changement de statut ultérieur.

En résumé, les pays/républiques/entités inclus dans les tableaux comparant ces trois groupes sont les suivants :

OCDE : Australie, Autriche, France, Allemagne, Islande, Irlande, Pays-Bas, Norvège, Espagne, et Suède. Sur tous les pays membres de l'OCDE, nous avons choisi d'inclure dans les tableaux ces 10 pays, représentatif d'un large choix de régions, de tailles et d'approches en ce qui concerne les BG et les CPM. Le texte cite parfois des exemples d'autre pays. Nous n'avons inclus aucun pays présidentiel, dans la mesure où l'étude du BG et du CPM n'est pas pleinement applicable dans leur cas. Dans les systèmes semi-présidentiels comme la France, l'accent est mis sur le Premier ministre et le gouvernement (et non sur l'administration du Président).

PECO : Bulgarie, République tchèque, Estonie, Hongrie, Lettonie, Lituanie, Pologne, Slovaquie et Slovénie. (La Roumanie n'a pas rempli le questionnaire et ne figure donc pas dans les tableaux).

Les Balkans occidentaux : Albanie, Bosnie-Herzégovine (État et Fédération de Bosnie-et-Herzégovine), Croatie, ancienne République yougoslave de Macédoine, Serbie-et-Monténégro (Communauté d'États et République du Monténégro) et Kosovo ; [la Serbie-et-Monténégro/Serbie et la BosnieHerzégovine/République serbe de Bosnie n'ont pas fourni d'informations et ne figurent donc pas dans les tableaux.]

\section{Mission et objectifs du Bureau du Gouvernement}

Les gouvernements et les administrations modernes sont complexes; ils constituent en réalité un ensemble de complexités multidimensionnelles. La notion de gouvernement englobe une variété presque infinie de thèmes, des droits de l'homme au développement économique, de l'environnement aux transports, sans oublier l'éducation, l'agriculture, la police et les retraites. Les gouvernements et les administrations traitent à court et long terme aussi bien les valeurs individuelles et les aspirations collectives que les questions locales et mondiales, les citoyens, les groupes, les régions, les minorités ethniques, les nations et la communauté internationale. Un gouvernement est tissé de plusieurs niveaux corrélés - municipaux, locaux, régionaux, nationaux et supranationaux. Dans les pays de l'Europe centrale et orientale, dans les Balkans occidentaux et dans l'ex-Union soviétique, les gouvernements doivent traiter tous ces aspects dans le contexte de la transition (nationale et économique) et le faire rapidement afin de répondre aux aspirations de leurs 
populations soucieuses de « rattraper» leur retard sur l'Ouest, tout en maintenant et en renforçant leurs propres cultures et institutions spécifiques.

La complexité des gouvernements et des administrations se vérifie également dans leur organisation, composée d'un grand nombre de ministères, eux-mêmes divisés en plusieurs secteurs, unités et autres organes décentralisés et déconcentrés, ainsi qu'en agences, commissions et autres représentations internationales. Les gouvernements démocratiques combinent des composantes variables soumises aux élections et autres remaniements périodiques, et des personnels plus permanents au service à la fois des citoyens et des politiciens. L'interaction entre ces composantes, chacune répondant à ses propres motivations et aspirations, est souvent loin d'être simple. C'est dire à quel point il est miraculeux que les gouvernements modernes ne dégénèrent pas plus souvent en véritable chaos ! Et de rappeler cette vérité de La Palice selon laquelle la coordination est la clé même d'un gouvernement efficace, à l'instar de n'importe quelle organisation complexe.

L'American Heritage Dictionary définit la «coordination» comme «la régulation de divers éléments dans une opération intégrée et harmonieuse ». Le Cambridge Dictionary définit quant à lui le verbe « coordonner » comme «faire fonctionner différentes choses efficacement comme un seul ensemble ». D'un point de vue plus opérationnel, Bordeau et Wasson, dans un article sur l'information et la communication (1997), définissent la «coordination» comme «l'action de gérer les corrélations entre les activités et les interdépendances parmi les acteurs ». Ces définitions méritent quelques remarques. Tout d'abord, le terme «coordination» tend à avoir des connotations positives, indiquées par l'emploi de mots tels que « harmonieux » et «efficace». Deuxièmement, on suppose implicitement que la coordination n'est pas une action autonome mais requiert gestion et régulation. Troisièmement, la «coordination » est généralement définie comme un processus plutôt que comme un résultat. Enfin, la coordination permet à des éléments et à des acteurs de conserver leur pluralité et leur différences, tout en poursuivant l'objectif d'obtenir des résultats harmonieux et efficaces.

Toutes les organisations grandes et complexes développent des moyens de coordination, souvent aux divers niveaux qui les composent. Le siège social de Coca-Cola à Atlanta, ou celui de l'ONU à New York, ou encore l'état-major de n'importe quelle organisation militaire constituent la forme la plus aboutie d"un système multiniveaux de coordination interne et entre unités, qui peuvent être organisés à l'échelle d'une région, d'un secteur ou de toute autre forme de regroupement. Quelles que soient l'importance et la complexité d'une organisation, la raison d'être, la justification et la «valeur ajoutée » de son siège ne trouvent leur véritable sens que dans le fait de gérer la coordination des décisions et de superviser le fonctionnement de l'organisation dans son ensemble. Aucun siège social ne saurait se substituer aux unités ou aux divisions, effectuer leur travail ni même les micro-gérer. Un siège social est là pour s'assurer, du mieux qu'il peut, que chaque société, filiale ou division effectue bien la tâche qui lui a été assignée, et qu'ensemble elles remplissent les objectifs collectifs.

L'analogie avec le gouvernement est évidente. Dans les régimes parlementaires ainsi que, dans une large mesure, les systèmes semi-présidentiels, le système décisionnel trouve sa forme la plus aboutie dans les sessions hebdomadaires du Conseil des ministres, présidées par le Premier ministre. Dans ces systèmes, ces sessions ont pour objectif d'accomplir les missions suivantes :

- Adopter une vision stratégique : définir les objectifs généraux du gouvernement, les classer par ordre de priorité et doter ces priorités en ressources ;

- Discuter des questions politiques et de communication auquel le gouvernement est confronté ;

- $\quad$ Prendre les décisions importantes au nom du gouvernement, en veillant à fédérer l'engagement de tous les ministres ;

- Prendre des décisions cohérentes : c'est-à-dire, des décisions qui sont légales, pour lesquelles les ressources financières et humaines ont été identifiées et dans lesquelles les différents intérêts des ministres ont été identifiés et conciliés ; et

- Endosser la responsabilité collective des réalisations du gouvernement.

La prise de décision dans les systèmes parlementaires démocratiques (y compris les systèmes semiprésidentiels) se fonde sur le double rôle joué par tous les membres du gouvernement. En général, les ministres endossent la responsabilité personnelle de leur propre domaine politique (finances, santé, agriculture, etc.) et, dans l'accomplissement de cette responsabilité, sont assistés de leur propre ministère. En 
outre, ils participent collectivement et régulièrement aux sessions du Conseil des ministres, à l'occasion desquelles ils discutent des propositions émanant des membres du gouvernement, prennent des décisions et sont tenus d'endosser la responsabilité collective aussi bien du programme gouvernemental que des décisions spécifiques. Dans l'exercice de leur rôle collectif, notamment dans leur représentation collective du gouvernement, les ministres sont aidés et soutenus par le BG.

La première mission du Premier ministre est dédiée au fonctionnement collectif du gouvernement, un rôle qu'il exerce habituellement en présidant les sessions du Conseil des ministres, puis dans un second temps, d'assurer le déroulement ordonné et logique du programme gouvernemental. Le Premier ministre a également la responsabilité de représenter le pays à l'étranger et de gérer la coalition. En sa qualité de président du CdM, le Premier ministre est assisté du BG. Généralement, le bureau politique ou «cabinet » du Premier ministre (CPM) fait également partie du BG, du moins d'un point de vue administratif, et les deux bureaux se partagent habituellement le même site, quoique, d'un point de vue fonctionnel, ils restent souvent distincts dans une certaine mesure.

En résumé, les responsabilités du BG dérivent directement des responsabilités collectives du gouvernement et du PM. Dès lors, l'unique but du BG est d'assister le Conseil des ministres et son Président dans leurs tâches de coordonner les activités de tous les ministères en vue de faciliter l'exécution efficace et efficiente de leurs devoirs collectifs. Le BG est donc essentiellement un organe de coordination au sein de l'administration. Très souvent, le BG doit remplir son rôle de coordination sans réellement occuper de rang hiérarchique officiellement supérieur aux ministères. Sa capacité d'exécuter son rôle de coordination ne dérive donc pas d'une quelconque aptitude à commander ou à ordonner aux ministères d'agir d'une certaine manière, mais de l'acceptation par tous les acteurs du système de l'importance de la coordination. Lorsque cette acceptation ne suffit pas, le BG requiert l'autorité du Premier ministre pour imposer une discipline collective au gouvernement.

\section{Outils pour accroître la cohérence des politiques}

Ces dernières années, le Service de la gestion publique de l'OCDE (PUMA) - Direction de la gouvernance publique et du développement territorial (GOV) depuis septembre 2002 - a mis en lumière le besoin croissant et la difficulté toujours plus grande des Membres de l'OCDE à maintenir une cohérence dans leurs politiques. Les raisons sont nombreuses et variées, au nombre desquelles on peut citer le besoin de gérer et d'optimiser l'efficacité de ressources limitées, et le changement rapide et continu qui requiert une certaine flexibilité ; l'interpénétration des domaines de politique internationale et intérieure qui exige de gérer la prise de décision à plusieurs niveaux ; l'explosion de l'information qui a multiplié le nombre d'acteurs dans l'arène politique et qui a favorisé la fragmentation des politiques. Dans une publication occasionnelle intitulée Renforcer la cohérence des politiques - Outils et tensions ${ }^{2}$, le PUMA a démontré que les questions d'organisation interne interfèrent également avec la cohérence, et remarqué que :

«Les gouvernements sont sujets à des tensions politiques divergentes, souvent incarnées par les intérêts conflictuels et sectoriels des ministères de tutelle, qui luttent entre eux pour obtenir des ressources financières, alors que le ministère du budget cherche de manière caractérisée à contrôler les dépenses. Dans certains pays, un certain degré de concurrence politique est délibérément maintenu entre les ministères de tutelle, afin de garantir un certain niveau de tension créative et d'inciter les ministères à remettre en question les conseils politiques. Tous les pays sont contraints de faire d'importants compromis entre des intérêts divergents au plus haut niveau. ».

Cette publication occasionnelle note cependant que les gouvernements comprennent mieux aujourd'hui que des politiques incohérentes renforcent le risque de duplication, de dépenses inefficaces, de services de faible qualité, d'objectifs difficilement réalisables et, enfin, de moyens de gouverner réduits. Néanmoins, le document avertit également qu'en dépit des bonnes raisons de renforcer la cohérence des politiques, il existe également des raisons de se montrer circonspect, compte tenu des réelles limites pratiques au-delà desquelles la cohérence ne peut véritablement être augmentée. En outre, il est important de s'assurer que les efforts pour obtenir une plus grande cohérence n'aboutissent pas à un contrôle excessif et à une perte conséquente de flexibilité et de créativité dans le système d'élaboration des politiques. 
L'examen des diverses expériences au sein des pays membres de l'OCDE a permis à la GOV d'identifier huit outils fondamentaux de cohérence. Après avoir noté que "l'outil principal de la cohérence est une prise de décision éclairée », les outils suivants sont répertoriés, sous réserve toutefois d'être adaptés à chaque système national avec toute la précaution et la sensibilité qui s'imposent :

- L'engagement de la direction politique est une condition préalable nécessaire à la cohérence, et un outil susceptible de la renforcer.

- Instaurer un cadre de politique stratégique permet d'avoir l'assurance que les différentes politiques sont compatibles avec les objectifs et les priorités du gouvernement.

- $\quad$ Les décideurs ont besoin d'une définition claire et d'une bonne analyse des questions, contenant des indications explicites des éventuelles contradictions.

- $\quad$ La mise en place de moyens centraux d'examen et de coordination est essentielle pour assurer une cohérence horizontale entre les politiques.

- $\quad$ Des mécanismes permettant d'anticiper, de détecter et de résoudre les conflits entre politiques dès les premières phases du processus permettent d'identifier les contradictions et de minimiser l'incohérence.

- Le processus décisionnel doit être organisé de manière à pouvoir réconcilier de manière efficace les priorités politiques et les impératifs budgétaires.

- $\quad$ Des procédures d'application et des mécanismes de suivi doivent être développés pour s'assurer que les politiques peuvent être ajustées à la lumière des progrès, des nouvelles informations et de l'évolution des circonstances.

- Une culture administrative qui favorise la coopération intersectorielle et un dialogue systématique entre les différentes communautés de politique contribue à renforcer la cohérence des politiques.

Les principaux acteurs censés recourir à ces outils sont la direction politique, les ministères, le BG et le CPM.

$\mathrm{Au}$ cours de ces douze dernières années, les travaux menés par Sigma sur les Bureaux de gouvernement en Europe centrale et orientale ainsi que dans les Balkans occidentaux sont parvenus à des conclusions très similaires sur le renforcement du système d'élaboration des politiques et sur le rôle du BG à cet égard. Depuis 1997, Sigma a réalisé des études détaillées sur les BG de sept pays de la région. En outre, depuis 1999, Sigma évalue pour le compte de la Commission européenne la performance des systèmes de politiques dans tous les PECO, sur la base d'un référentiel commun. L'ensemble de ces évaluations parviennent à la conclusion principale que l'utilisation efficace de ces outils pour promouvoir la cohérence des politiques exige des moyens corrélés dans les ministères de tutelle et le BG.

Dans les ministères de tutelle, l'utilisation efficace des outils passe par des procédures et des capacités visant à élaborer des politiques et des propositions de loi de grande qualité. Plus particulièrement, ces procédures doivent comprendre des consultations entre les ministères et les parties prenantes, et être assorties de moyens permettant de fonder les propositions sur des analyses valides basées sur l'évaluation ex-ante des impacts des propositions et sur l'évaluation à posteriori périodique des résultats.

Le BG joue un rôle central en termes de spécification, de promotion et d'utilisation de ces outils pour renforcer la cohérence des politiques. Il lui appartient d'établir, de mettre à jour et de faire exécuter les procédures applicables en matière de préparation et de soumissions au CdM. Il doit être en mesure d'assurer la coordination et de fournir des analyses et des conseils pour faciliter et garantir un flux d'informations de haute qualité lors des sessions du CdM et doit veiller à ce que la plupart des conflits, notamment de nature technique, soient résolus avant de soumettre les questions au CdM.

Les fonctions et les activités des Bureaux de gouvernement dans les pays membres de l'OCDE, les PECO et les pays des Balkans occidentaux sont plus amplement comparées dans la prochaine section de cette publication.

\section{Principales fonctions du BG : huit axes de coordination}

Il est utile de considérer toutes les fonctions des BG comme reliées à la coordination. En règle générale, le BG est rarement responsable directement d'un domaine de politique spécifique (à quelques exceptions près). En revanche, le BG est un coordonnateur, dont la tâche consiste à, si l'on reprend la définition donnée par le Cambridge Dictionary «faire fonctionner différentes choses efficacement comme un seul ensemble ». Dans cette section, les fonctions communes aux BG de différents pays sont abordées sous huit intitulés, chacun désignant un aspect de la coordination. Dans de nombreux cas, le BG partage certaines de ces responsabilités 
avec le CPM, mais il serait contre-productif et inutile d'essayer de les distinguer en permanence, c'est pourquoi la présente publication a pris le parti d'englober également le CPM dans la définition du BG. Les huit axes de coordination abordés ci-après sont les suivants :

1 Coordination de la préparation des sessions du gouvernement ;

2 Coordination de la conformité juridique ;

3 Coordination de la préparation du programme et des priorités gouvernementales et de leur dotation au budget ;

4 Coordination du contenu des propositions de politique pour le CdM ;

5 Coordination des communications, p. ex. communiqués de presse ;

6 Coordination du suivi de l'exécution gouvernementale ;

7 Coordination des échanges avec les autres instances de l'État (Président, Parlement); et

8 Coordination des priorités stratégiques horizontales spécifiques.

\subsection{Coordination de la préparation des sessions du gouvernement}

En matière de coordination, la contribution la plus courante du BG passe par la gestion logistique en vue de préparer les sessions du CdM. Cette fonction se retrouve dans tous les BG des pays de l'OCDE, des PECO et des pays des Balkans occidentaux. Il s'agit d'une fonction essentiellement technique, garante du succès du $\mathrm{CdM}$ dans la mesure où elle concerne la gestion du processus décisionnel en établissant des règles et en veillant à leur application. En tant que processus, la coordination des politiques est vouée à créer des conditions - en particulier, des procédures normalisées au sein de l'appareil étatique - en vertu desquelles elle peut être obtenue aussi bien au sein qu'à travers différents secteurs politiques. Comme nous le montrerons ci-après, la coordination procédurale ne suffit pas à garantir que les sessions du CdM donnent des résultats de grande qualité ; néanmoins, elle est absolument nécessaire à une prise de décision de haute qualité. La coordination procédurale présente l'avantage supplémentaire d'être relativement facile à améliorer avec des techniques faciles à appliquer et qui ne sont pas controversées.

À une ou deux exceptions près, tout les CdM étudiés ici tiennent régulièrement des sessions hebdomadaires essentiellement axées sur les questions préparées par les ministères et envoyées au BG pour révision et inscription à l'ordre du jour. Le soutien logistique fournit par le BG englobe les principales activités suivantes :

\subsubsection{Délais de soumission et de diffusion des documents pour les sessions}

Si les documents n'arrivent pas à temps, les ministres risquent de ne pas pouvoir les étudier ni de consulter leurs représentants avant la session du CdM. Ainsi, dans la plupart des pays, il incombe au BG de veiller au respect des délais. Par exemple, en Autriche, les documents sont diffusés cinq jours avant la session pour permettre des consultations préalables (par exemple avec les partis de la coalition). En Belgique, les documents doivent être transmis au BG le lundi précédant la session du vendredi, et en Irlande, ils doivent être diffusés trois jours avant la réunion. En Bulgarie, en République tchèque et en Estonie, les documents doivent être diffusés sept jours avant les réunions. Généralement, la norme pour distribuer les documents dans la plupart des pays semble être de trois à sept jours avant la réunion .

Dans la majorité des pays, l'ordre du jour pour chaque session est préparé par le Secrétaire Général et approuvé par le Premier ministre. Pour chaque session hebdomadaire, il existe habituellement des règles qui spécifient la date butoir à laquelle les documents doivent être soumis au BG pour être programmés pour une réunion spécifique. Ce délai prend en compte le nombre de jours qu'il faut au BG pour passer en revue les documents, pour tenir toutes les réunions potentiellement nécessaires pour revoir les questions avant la session du CdM (par exemple, comités de «filtre » du CdM, réunion hebdomadaire des secrétaires d'État). Par exemple, dans l'ancienne République yougoslave de Macédoine, les questions soumises aux sessions du gouvernement doivent être transmises au BG 15 jours avant la session dès lors qu'elles concernent des lois organiques ou d'autres sujets importants, sinon elles doivent être communiquées 8 jours à l'avance. 
Tableau 1. Préparation des sessions du gouvernement

\begin{tabular}{|l|c|c|}
\hline \multicolumn{1}{|c|}{ Pays membres de l'OCDE } & $\begin{array}{c}\text { Délai consenti aux ministères pour } \\
\text { soumettre les questions au BG avant la } \\
\text { session du CdM }\end{array}$ & $\begin{array}{c}\text { Délai pour distribuer les documents aux } \\
\text { ministères avant la date } \\
\text { de session du CdM }\end{array}$ \\
\hline Australie & 5 jours & 5 jours \\
\hline Autriche & 5 jours & 5 jours \\
\hline France & 5 jours & 3 jours \\
\hline Allemagne & 14 jours & 7 jours \\
\hline Islande & Aucun délai & 3 jours \\
\hline Irlande & 3 jours & 7 jours \\
\hline Pays-Bas & 7 jours & 2 jours \\
\hline Norvège & 2 jours & 4 jours \\
\hline Espagne & 4 jours & Aucun délai \\
\hline Suède & 1 jour & \\
\hline
\end{tabular}

\begin{tabular}{|l|c|c|}
\hline \multicolumn{1}{|c|}{ PECO } & $\begin{array}{c}\text { Délai consenti aux ministères pour } \\
\text { soumettre les questions au BG avant la } \\
\text { session du CdM }\end{array}$ & $\begin{array}{c}\text { Délai pour distribuer les documents aux } \\
\text { ministères avant la date } \\
\text { de session du CdM }\end{array}$ \\
\hline Bulgarie & 10 jours & 7 jours \\
\hline République Tchèque & 7 jours & 7 jours \\
\hline Estonie & 14 jours & 7 jours \\
\hline Hongrie & 4 jours & 4 jours \\
\hline Lettonie & 2 jours & 3 jours \\
\hline Lituanie & $20-28$ jours & 5 jours \\
\hline Pologne & 5 jours & Aucun délai \\
\hline Slovaquie & Aucun délai & 4 jours \\
\hline Slovénie & 4 jours & \\
\hline
\end{tabular}

\begin{tabular}{|l|c|c|}
\hline \multicolumn{1}{|c|}{ Pays des Balkans occidentaux } & $\begin{array}{c}\text { Délai consenti aux ministères pour } \\
\text { soumettre les questions au BG avant la } \\
\text { session du CdM }\end{array}$ & $\begin{array}{c}\text { Délai pour distribuer les documents aux } \\
\text { ministères avant la date } \\
\text { de session du CdM }\end{array}$ \\
\hline Albanie & Aucun délai & 2 jours \\
\hline Bosnie-Herzégovine (au niveau de l'état) & Aucun délai & 3 jours \\
\hline Bosnie-Herzégovine (Fédération) & 7 à 2 jours & 2 jours \\
\hline Croatie & Aucun délai précis mais procédure \\
régulière pour examen préalable & 7 jours \\
\hline Ancienne République yougoslave de & 15 jours & Dès leur réception \\
\hline Serbie et Monténégro (Fédération) & 5 jours & 7 jours \\
\hline Serbie-Monténégro / Monténégro & 9 jours & 5 jours \\
\hline Serbie-Monténégro / Kosovo & 5 jours & \\
\hline
\end{tabular}


Beaucoup de pays ont recours à des commissions ou à des comités ministériels pour examiner les questions et résoudre les conflits avant de les soumettre au CdM. Auquel cas, les questions doivent arriver à temps pour être d'abord programmées dans les comités, et ainsi prévoir leur diffusion en session plénière du CdM. Au Canada, les documents doivent être soumis cinq jours avant la réunion du comité approprié, pour être distribués aux membres au moins trois jours à l'avance. Les questions sont alors programmées pour une session plénière du $\mathrm{CdM}$, après avoir été approuvées par le comité, et un délai doit être prévu pour leur distribution ultérieure.

Un certain nombre de PECO et de pays des Balkans occidentaux, comme la Roumanie, la République tchèque, la Slovaquie, et la Bosnie-Herzégovine (État), sont confrontés au problème du trop grand nombre de questions soumises à la dernière minute et directement traitées en session de $\mathrm{CdM}$. La plupart des systèmes gèrent les questions de dernière minute, mais cela peut poser problème lorsqu'elles occupent une place importante dans l'ordre du jour et lorsqu'elles concernent des aspects politiques importants exigeant un examen plus approfondi. Il est crucial que le BG développe des méthodes pour contrôler cette tendance afin de prévenir (ou de limiter) les situations dans lesquelles des décisions importantes sont prises sans réflexion suffisante. Par exemple, le Portugal a institué comme règle que seul le Premier ministre peut soumettre des questions qui n'ont pas été préalablement inscrites à l'ordre du jour de la session. Dans beaucoup d'autre pays, la règle est que les ministres peuvent inscrire des questions à l'ordre du jour à la dernière minute uniquement après autorisation préalable du Premier ministre.

Le problème majeur ici est de savoir si oui ou non, et dans quelle mesure, les ministres sont «autorisés » à soumettre directement des documents lors des sessions, au mépris de tout délai. Dans un tel cas de figure, les propositions contournent le processus de résolution des conflits additionnels préalable à la session, via par exemple les comités ministériels et la réunion hebdomadaire des secrétaires d'état (s'ils existent), ainsi que l'examen préalable du BG (voir ci-dessous). La plupart des systèmes tolèrent certaines exceptions, mais idéalement de telles exceptions devraient rester rares et faire l'objet de procédures, par exemple elles doivent être urgentes et requérir l'autorisation du Premier ministre ou du Secrétaire Général. Lors de l'analyse finale, la seule personne généralement apte à contrôler la soumission de questions non finalisées est le Premier ministre. Ces dernières années, Sigma cite dans ses rapports la Bulgarie comme un bon exemple de gouvernement dans lequel le Premier ministre exerce son pouvoir pour contrôler de manière particulièrement efficace la soumission de questions de dernière minute. 
Tableau 2. Respect des règles

\begin{tabular}{|l|c|c|}
\hline \multicolumn{1}{|c|}{ Pays membres de l'OCDE } & $\begin{array}{c}\text { Les délais de soumission sont-ils } \\
\text { respectés en règle générale ? }\end{array}$ & $\begin{array}{c}\text { Les ministres soumettent-ils des } \\
\text { questions non programmées }\end{array}$ \\
\hline Australie & Oui & Rarement \\
\hline Autriche & Avec de nombreuses exceptions & Rarement \\
\hline France & À quelques exceptions & Non \\
\hline Allemagne & Oui & Non \\
\hline Islande & Avec de nombreuses exceptions & Souvent \\
\hline Irlande & Oui & Rarement \\
\hline Pays-Bas & Oui & Rarement \\
\hline Norvège & Oui & Rarement \\
\hline Espagne & Oui & Rarement \\
\hline Suède & Avec de nombreuses exceptions & \\
\hline
\end{tabular}

\begin{tabular}{|l|c|c|}
\hline \multicolumn{1}{|c|}{ PECO } & $\begin{array}{c}\text { Les délais de soumission sont-ils } \\
\text { respectés en règle générale ? }\end{array}$ & $\begin{array}{c}\text { Les ministres soumettent-ils des } \\
\text { questions non programmées }\end{array}$ \\
\hline Bulgarie & Oui & Non \\
\hline République Tchèque & Avec de nombreuses exceptions & Ravent \\
\hline Estonie & Avec de nombreuses exceptions & Rarement \\
\hline Hongrie & Oui & Rarement \\
\hline Lettonie & Avec de nombreuses exceptions & Rarement \\
\hline Lituanie & Avec de nombreuses exceptions & Rarement \\
\hline Pologne & Oui & Souvent \\
\hline Slovaquie & Avec de nombreuses exceptions & Rarement \\
\hline Slovénie & Oui & \\
\hline
\end{tabular}

\begin{tabular}{|l|c|c|}
\hline \multicolumn{1}{|c|}{ Pays des Balkans occidentaux } & $\begin{array}{c}\text { Les délais de soumission sont-ils } \\
\text { respectés en règle générale ? }\end{array}$ & $\begin{array}{c}\text { Les ministres soumettent-ils des } \\
\text { questions non programmées }\end{array}$ \\
\hline Albanie & Oui & Rarement \\
\hline Bosnie-Herzégovine (au niveau de l'état) & Non & Souvent \\
\hline Bosnie-Herzégovine (Fédération) & Avec de nombreuses exceptions & Rarement \\
\hline Croatie & Oui & Souvent \\
\hline Ancienne République yougoslave de & Avec de nombreuses exceptions & Rarement \\
\hline Serbie et Monténégro (Fédération) & Oui & Rarement \\
\hline Serbie-Monténégro / Monténégro & Oui & Rarement \\
\hline Serbie-Monténégro / Kosovo & Avec de nombreuses exceptions & \\
\hline
\end{tabular}


Les réponses au questionnaire reportées dans ces tableaux suggèrent que l'inobservation des délais est une pratique relativement courante, mais que la soumission directe au $\mathrm{CdM}$ de questions qui n'ont pas été préalablement examinées ni programmées par le BG reste peu répandue. Dans le passé, Sigma a souvent constaté que de telles pratiques étaient en réalité assez courantes dans les pays de l'Europe centrale et orientale ainsi que dans les Balkans occidentaux, et avait formulé des observations négatives à ce sujet. Les résultats rapportés ici suggèrent certaines améliorations dans ces pratiques et montrent que les BG dans la région sont désormais davantage en mesure de contrôler le processus de préparation des sessions du CdM. Il ne faut pas non plus écarter la possibilité que les gouvernements ont tendance à se noter de manière trop positive.

\subsubsection{L'ordre du jour des sessions du CdM}

La programmation anticipée de l'ordre du jour du CdM est fondamentale. Dans beaucoup de pays, les questions inscrites à l'ordre du jour dépendent presque totalement des propositions en provenance des ministères avant une certaine date. L'Irlande est un exemple typique de cette approche : «L'ordre du jour est préparé par le Secrétaire du Gouvernement et répertorie dans une liste toutes les notes de service reçues des Ministres ». D'autres pays s'essaient à une planification anticipée. En Estonie, en Lettonie et en SerbieMonténégro/Kosovo, par exemple, lors de leur réunion hebdomadaire, les secrétaires d'État, qui déterminent les questions à l'ordre du jour de la semaine en cours, préparent également l'ordre du jour de la semaine suivante.

En France, un système encore plus élaboré permet une meilleure planification anticipée de l'ordre du jour. L'ordre du jour est dérivé du programme de travail du gouvernement, qui est habituellement préparé chaque semestre par le Cabinet du Premier ministre (CPM) et par le BG (Secrétariat général du Gouvernement). Des ordres du jour provisoires pour les huit sessions du Conseil des Ministres à venir sont préparés le vendredi matin lors de deux réunions successives. La première de ces réunions est interne au $\mathrm{BG}$; la seconde est une réunion conjointe du CPM et du $\mathrm{BG}$.

Cette structure de l'ordre du jour du CdM peut également être employée pour rationaliser les réunions et consacrer plus de temps aux questions importantes ou controversées. En France, par exemple, l'ordre du jour se divise en trois volets : le premier est consacré aux projets de lois qui ne donnent généralement lieu à aucun débat dans la mesure où toutes les questions ont été validées ; le deuxième est dédié aux nominations, lesquelles ne suscitent habituellement aucun débat ; seul le troisième volet de l'ordre du jour aborde les questions de politique et de communication qui exigent un débat et la soumission de points de vue. Ce troisième volet constitue clairement le pivot des sessions. Aux Pays-Bas et en Allemagne, l'ordre du jour se divise en deux parties, que les questions évoquées suscitent au non un débat.

En Roumanie, la première partie de l'ordre du jour hebdomadaire de la session gouvernementale est consacrée à l'examen des questions stratégiques. C'est uniquement après avoir examiné ces questions que la réunion passe à l'approbation des documents juridiques et des autres questions exigeant une décision formelle. L'inscription des questions à la première partie de l'ordre du jour se fait sur décision du Premier ministre après proposition des ministres, qui préparent généralement des documents de consultation sur leurs questions. Dernièrement, l'ancienne République yougoslave de Macédoine a également commencé à utiliser un ordre du jour en trois volets qui s'inspire du modèle français, et les ministres reconnaissent que ce système a amélioré la conduite et l'efficacité des sessions. Diviser l'ordre du jour en plusieurs parties peut aider les ministres à concentrer leur préparation et leur attention sur les questions importantes ; une approche d'autant plus utile lorsque l'ordre du jour tend à être long, et lorsqu'une quantité considérable de documents s'y rapporte.

\subsubsection{Examen des documents pour s'assurer de l'exhaustivité des dossiers et de l'observation des procédures de consultation}

La plupart des pays se sont dotés de procédures détaillées qui réglementent la préparation des documents pour le CdM, y compris les exigences de documentation des analyses. Ces règles portent également sur les étapes que les ministères à l'origine de propositions doivent suivre pour veiller à ce que les autres ministères intéressés, et parfois certains groupes extérieurs, soient adéquatement consultés avant de soumettre la proposition au BG pour programmation. La qualité et la portée de telles consultations interministérielles et autres figurent parmi les facteurs qui contribuent le plus à la cohérence des politiques. Les consultations doivent englober toutes les parties intéressées, se dérouler dès les premières phases du processus de 
préparation, et accorder aux parties consultées suffisamment de temps pour considérer les questions et y répondre. Ces consultations doivent être ouvertes et informelles. Si ces consultations sont convenablement menées, il y a de grandes chances de pouvoir corriger les éléments contradictoires ou illogiques contenus dans les politiques et les projets de lois avant de soumettre les propositions aux sessions du CdM. Les consultations relèvent de la responsabilité des ministères, et il appartient généralement au BG de vérifier que de telles consultations ont été menées avec toutes les parties intéressées, et que leurs opinions ont été raisonnablement entendues et considérées.

Puisque ces procédures sont normalement spécifiées dans les règles, le BG doit, au minimum, vérifier qu'elles ont été suivies. Cependant, ce n'est pas toujours aussi simple. Par exemple, dans de nombreux cas, les règles spécifient que certains ministères (par exemple, des finances et/ou de la justice) doivent toujours être consultés, ou qu'un secrétariat législatif doit être consulté sur tous les actes juridiques. En outre, ces mêmes règles indiquent souvent que «tous les autres ministères compétents devraient également être consultés ». L'Australie offre un bon exemple d'une telle approche. « Le guide du gouvernement exige que les ministres et les fonctionnaires des ministères dans l'intérêt de la proposition doivent avoir l'opportunité suffisante de contribuer à son élaboration et de résoudre les divergences avant sa soumission au BG. La proposition doit être assortie de commentaires de coordination de tous les ministères ayant un intérêt ». Peut-on toujours compter sur les ministères pour identifier correctement les parties à consulter ? Qu'en est-il de la réponse réservée par le ministère à l'origine de la proposition aux commentaires des autres ministères ? Les a-t-il considérés sérieusement ou, s'il les a rejetés, ce rejet a-t-il été motivé sur une base solide ? Le BG, dans son devoir de garantir une prise de décision éclairée, devrait être autorisé à vérifier tous ces aspects de la préparation et des consultations entre les ministères. Un rôle utile, donc, d'autant plus si le BG est impliqué dès les premières étapes du processus et reçoit une bonne documentation sur le processus de consultation.

En règle générale, le $\mathrm{BG}$ est le garant des règles relatives à la finalisation des dossiers. Par exemple, il vérifie si toutes les signatures requises sont apposées, si les exposés des motifs sont bien inclus, et si la législation dérivée est incluse avec la législation primaire (lorsque ces questions sont exigées par les règles). La question principale est la suivante : en cas de dossier incomplet, en cas de questions cruciales manquantes, en cas de ministères compétents non consultés, etc., le BG peut-il retourner le dossier au ministère et refuser de le programmer jusqu'à ce qu'il soit jugé complet ?

Dans presque tous les pays des Balkans occidentaux, les règles autorisent le BG à renvoyer les questions aux ministères uniquement pour des raisons techniques, formelles, etc., par exemple en cas de signatures ou de documents requis manquants. Malheureusement, ces prérogatives ne portent pas sur l'utilité et l'exhaustivité des consultations. Par exemple, dans beaucoup de ces pays, les règles spécifient que le ministère à l'origine d'une proposition doit inclure un document qui indique les personnes consultées et les résultats de ces consultations. Le BG est autorisé à renvoyer la proposition au ministère si ce document fait défaut. Cependant, le BG n'est pas habilité à juger si tous les ministères compétents ont bien été consultés ni à renvoyer alors les documents s'il estime qu'il y a des lacunes. Dans de nombreux cas, même lorsque le BG est formellement investi du pouvoir de renvoyer des propositions, il peut manquer de moyens ou d'autorité pour faire exécuter sa décision dans la pratique. Dans le passé, Sigma observait généralement dans ses évaluations que la situation dans les PECO était similaire à ce cas de figure. Cependant, les questionnaires indiquent que dans cinq PECO sur neuf, le BG est désormais habilité à se prononcer sur l'adéquation des politiques et du programme et à renvoyer ces questions aux ministères pour un travail complémentaire en cas de teneur inadéquate.

Enfin, si les ministres veulent faire passer une proposition, ils peuvent souvent consulter le Premier ministre et faire inscrire la proposition à l'ordre du jour à la dernière minute. Le BG a donc besoin du soutien du PM lorsqu'il renvoie des propositions incomplètes aux ministères, il risquerait autrement de perdre un outil particulièrement utile au maintien de l'intégrité du système décisionnel. 
Tableau 3. Motifs du retour des questions aux ministères pour un travail complémentaire

\begin{tabular}{|l|c|c|}
\hline \multicolumn{1}{|c|}{ Pays membres de l'OCDE } & $\begin{array}{c}\text { Le BG peut renvoyer des questions aux } \\
\text { ministères pour des motifs techniques }\end{array}$ & $\begin{array}{c}\text { Le BG peut renvoyer des questions aux } \\
\text { ministères pour des motifs politiques }\end{array}$ \\
\hline Australie & Oui & Oui \\
\hline Autriche & Oui & Oui \\
\hline France & Oui & Certaines (résolue par le BG par d'autres \\
\hline Allemagne & Oui & Oui \\
\hline Islande & Certaines & Oui \\
\hline Irlande & Aucune autorité pour renvoyer des & Non autorité pour renvoyer des \\
\hline Pays-Bas & Aucune autorité pour renvoyer des & Aucune autorité pour renver des \\
\hline Norvège & Oui & Oui \\
\hline Espagne & Oui & Oui \\
\hline Suède & Oui & \\
\hline
\end{tabular}

\begin{tabular}{|l|c|c|}
\hline \multicolumn{1}{|c|}{ PECO } & $\begin{array}{c}\text { Le BG peut renvoyer des questions aux } \\
\text { ministères pour des motifs techniques }\end{array}$ & $\begin{array}{c}\text { Le BG peut renvoyer des questions aux } \\
\text { ministères pour des motifs politiques }\end{array}$ \\
\hline Bulgarie & Oui & Non \\
\hline République Tchèque & Oui & Oui \\
\hline Estonie & Oui & Non \\
\hline Hongrie & Oui & Oui \\
\hline Lettonie & Oui & Oui \\
\hline Pologne & Oui & Oui \\
\hline Slovaquie & Oui & Non \\
\hline Slovénie & Oui & Non \\
\hline
\end{tabular}

\begin{tabular}{|l|c|c|}
\hline \multicolumn{1}{|c|}{ Pays des Balkans occidentaux } & $\begin{array}{c}\text { Le BG peut renvoyer des questions aux } \\
\text { ministères pour des motifs techniques }\end{array}$ & $\begin{array}{c}\text { Le BG peut renvoyer des questions aux } \\
\text { ministères pour des motifs politiques }\end{array}$ \\
\hline Albanie & Oui & Oui \\
\hline Bosnie-Herzégovine (au niveau de l'état) & Oui & Non \\
\hline Bosnie-Herzégovine (Fédération) & Oui & Non \\
\hline Croatie & Oui & Non \\
\hline Ancienne République yougoslave de & Oui & Oui \\
\hline Serbie et Monténégro (Fédération) & Oui & Non \\
\hline Serbie-Monténégro / Monténégro & Oui & Oui \\
\hline Serbie-Monténégro / Kosovo & Ouis prérogative préns le \\
\hline
\end{tabular}


La coordination de la conformité juridique des projets de lois est étroitement liée à la préparation des réunions du CdM. En Europe continentale, et plus particulièrement en Europe centrale et orientale ainsi que dans les Balkans occidentaux, le CdM joue un rôle crucial dans le processus d'élaboration des lois ; tous les documents législatifs soumis au CdM pour décision doivent être soigneusement examinés pour s'assurer qu'ils répondent aux exigences légales du pays. Ces exigences portent traditionnellement sur trois axes : conformité avec la Constitution, conformité avec les autres lois et réglementations, et conformité avec le style de rédaction juridique. Pour les pays souhaitant rejoindre l'UE, la conformité avec l'acquis communautaire constitue désormais un quatrième axe d'exigence.

La plupart des BG dans les PECO et les pays des Balkans occidentaux dirigent plus particulièrement leurs efforts sur l'examen juridique, alors que seuls quelques pays de l'OCDE s'y astreignent. Les BG des pays de l'OCDE voient rarement dans cet examen une fonction centrale. Par exemple, sur les 25 pays membres de l'OCDE ayant rempli un profil PUMA sur leurs centres gouvernementaux en 1996, seul un ou deux pays avaient énuméré la révision juridique comme une des fonctions de leur Bureau de gouvernement. (Le dernier questionnaire en date donne une vision quelque peu différente, mais la nature des réponses suggère que cette question a pu avoir été mal comprise, un point qui devra être plus amplement étudié.) Dans le cas des profils des Centres de gouvernement $(\mathrm{CdG})$ préparés par Sigma pour les PECO, tous les pays ont mentionné la révision juridique comme une des fonctions du BG. Dans les pays de l'ancienne Yougoslavie, d'autre part, il est habituel d'observer l'instauration d'un conseil juridique distinct et indépendant chargé de procéder à la révision juridique. Dans beaucoup de pays, que ce soit les pays membres de l'OCDE ou les PECO, le BG n'est pas le seul organe administratif chargé d'examiner la conformité juridique. Par exemple, en Slovaquie et en République tchèque, la conformité juridique est vérifiée par le $\mathrm{BG}$ et le Conseil législatif, et en Estonie à la fois par le BG et le Ministère de la justice.

En règle générale, cette fonction n'est pas un problème en soi, et s'exerce harmonieusement dans la plupart des PECO et des pays des Balkans occidentaux, que ce soit au sein du BG ou dans un conseil indépendant. Dans ses évaluations, il est rare que Sigma suggère de réformer cette fonction. Cependant, certaines questions connexes méritent considération. Le problème le plus important — du point de vue de la cohérence des politiques - est que l'attention portée sur la conformité juridique dans les PECO et les pays des Balkans occidentaux se substitue souvent aux préoccupations relatives au contenu politique des propositions de lois. Ce problème se pose non seulement au niveau des efforts quasiment exclusifs que le BG consacre aux aspects techniques/juridiques, mais également au niveau de la phase de préparation et de consultation entre les ministères. Par exemple, il est relativement courant de voir les ministères dans les pays des Balkans occidentaux et les PECO procéder directement à la rédaction des lois, avant d'en avoir clarifié et réglé les implications politiques et exécutoires. Dans de nombreux cas, les consultations interministérielles ne sont également sollicitées qu'une fois les propositions pleinement rédigées en textes de loi. Par conséquent, les consultations sont souvent plutôt formelles et font peser des pressions implicites sur les parties consultées pour commenter les questions techniques et rédactionnelles, au détriment des questions de fond sur la politique et ses coûts/avantages. Pour cette raison, les efforts considérables de réforme du BG - par exemple, en Lettonie, en Lituanie, et dans l'ancienne République yougoslave de Macédoine — doivent en partie s'employer à renforcer le rôle du BG en termes d'analyse et de coordination des politiques.

\subsection{Coordination de la préparation du programme gouvernemental (priorités stratégiques, programmes de travail annuels) et de leur dotation au budget}

La planification - à la fois stratégique et pour les travaux à accomplir - et la gestion des articulations dynamiques entre elles relèvent des responsabilités fondamentales du gouvernement et du Premier ministre. Un cadre stratégique, comme suggéré par le PUMA, est un outil central pour garantir la cohérence des politiques. Dans les démocraties parlementaires, la définition des objectifs stratégiques du gouvernement est une responsabilité politique, souvent reflétée dans les déclarations du gouvernement au parlement. D'autre part, la préparation des plans, l'analyse requise pour soutenir ces plans, et les activités nécessaires à leur mise en œuvre sont des tâches qui relèvent de l'administration. Alors que les ministères préparent les idées et analysent les informations dans leurs différents domaines, le BG a la responsabilité de coordonner les documents et d'assister le gouvernement dans la définition des priorités parmi les demandes concurrentes sur les ressources budgétaires et autres. 
Tableau 4. Capacité de planification stratégique et du travail pour le CdM

\begin{tabular}{|l|c|c|}
\hline \multicolumn{1}{|c|}{ Pays membres de l'OCDE } & Capacité de planification stratégique & Capacité de planification annuelle du \\
\hline Australie & Dans le CPM & Dans le BG \\
\hline Autriche & Non & Non \\
\hline France & Dans le BG & Dans le BG \\
\hline Allemagne & Dans le BG & Dans le BG \\
\hline Islande & Non & Dans le CPM \\
\hline Irlande & Dans le CPM & Dans le BG \\
\hline Pays-Bas & Dans le CPM & Don le CPM et le BG \\
\hline Espagne & Dans le BG & Dans le BG \\
\hline Suède & Aucune (doit bientôt être instaurée) & Dans \\
\hline
\end{tabular}

\begin{tabular}{|l|c|c|}
\hline \multicolumn{1}{|c|}{ PECO } & Capacité de planification stratégique & Capacité de planification annuelle du \\
\hline Bulgarie & Dans le BG & Dans le BG \\
\hline République Tchèque & Dans le BG & Dans le BG \\
\hline Estonie & Non & Dans le BG \\
\hline Hongrie & Dans le BG & Dans le BG \\
\hline Lettonie & Dans le BG & Dans le BG \\
\hline Lituanie & Dans le BG & Dans le BG \\
\hline Pologne & Dans le BG \\
& $\begin{array}{c}\text { Dans une instance distincte directement } \\
\text { rattachée au P.M. }\end{array}$ \\
\hline Slovaquie & Dans le BG & Dans le BG \\
\hline Slovénie & Non & Dans le BG \\
\hline
\end{tabular}

\begin{tabular}{|l|c|c|}
\hline \multicolumn{1}{|c|}{ Pays des Balkans occidentaux } & Capacité de planification stratégique & Capacité de planification annuelle du \\
\hline Albanie & Dans le BG & Non \\
\hline Bosnie-Herzégovine (au niveau de l'état) & Non & Dans le BG \\
\hline Bosnie-Herzégovine (Fédération) & Non & Dans le BG \\
\hline Croatie & Dans le BG & Dans le BG \\
\hline Ancienne République yougoslave de & Dans le BG & Dans le BG \\
\hline Serbie et Monténégro (Fédération) & Non & Dans le BG \\
\hline Serbie-Monténégro / Monténégro & Non & Non \\
\hline Serbie-Monténégro / Kosovo & Non & \\
\hline
\end{tabular}




\subsubsection{Planification stratégique}

Généralement, le rôle du BG en matière de planification stratégique comprend les tâches suivantes :

- $\quad$ Veiller à ce que les délibérations du gouvernement sur ses priorités stratégiques tiennent le plus largement compte de la situation globale économique, politique et sociale ;

- $\quad$ S'assurer que les priorités stratégiques sont harmonisées avec les autres documents stratégiques du gouvernement, tels que les stratégies d'adhésion à l'UE, économiques et budgétaires, et les autres stratégies fondamentales de politique et de réforme ;

- $\quad$ S'assurer que le processus de préparation du budget tient compte des priorités stratégiques et les reflète ;

- S'assurer que les programmes de travail des ministères reflètent les priorités stratégiques du gouvernement ;

- $\quad$ S'assurer que le Premier ministre est régulièrement instruit des nouveaux développements affectant les priorités stratégiques et le programme de travail annuel ainsi que des réponses ou des modifications possibles le cas échéant.

Dans la plupart des pays de l'OCDE, il appartient aux BG d'assurer la planification stratégique. Par exemple, en Autriche, une des fonctions du BG consiste à «formuler les politiques gouvernementales générales, en particulier l'orientation générale des politiques économiques». Au Canada, le BG «propose au Premier ministre d'assurer la gestion stratégique des questions gouvernementales ainsi que la planification globale et la définition des priorités ». En Suisse, tous les quatre ans, le Bureau du Gouvernement «prépare le programme gouvernemental pour la législature suivante, en collaboration avec les ministères. Ce «programme pour la durée du mandat » met en lumière les principaux problèmes et présente des solutions concrètes sous forme de déclaration » du CdM.

En revanche, dans les PECO et les pays des Balkans occidentaux, les BG ne disposent que de très peu de moyens pour coordonner la préparation des plans stratégiques ${ }^{3}$. Dernièrement, certains pays ont pris des mesures pour renforcer leurs moyens stratégiques, comme par exemple la Lettonie, la Lituanie, la Pologne et l'ancienne République yougoslave de Macédoine. Récemment, dans le cadre d'une réforme en profondeur de son BG, le Gouvernement de l'ancienne République yougoslave de Macédoine a arrêté la décision d'instaurer une unité de planification stratégique au sein du BG et prend désormais des mesures pour établir un processus de planification exhaustive corrélant les priorités budgétaires aux priorités stratégiques. Des développements semblables sont observés en Lettonie et en Lituanie. Le Tableau 4 prouve en réalité l'existence de certaines ressources de planification stratégique dans sept des neuf PECO ayant répondu au questionnaire. La Roumanie, qui n'a pas répondu, ne dispose pas de telles ressources. Les informations recueillies par Sigma lors de sa dernière campagne d'évaluations sur la coordination des politiques (2000) tendraient à suggérer que ces ressources restent en général insuffisantes dans la plupart des PECO.

\subsubsection{Planification annuelle du travail}

La plupart des gouvernements adoptent une ou plusieurs formes de planification de travail, généralement sur une base annuelle. Les programmes peuvent être axés sur l'ensemble des questions planifiées par les ministères pour le CdM, ou seulement sur certains aspects, comme par exemple un plan législatif. Dans presque tous les cas, la préparation de tels plans incombe au BG, et il ressort du Tableau 4 que presque tous les pays disposent des moyens nécessaires pour préparer des programmes de travail annuels.

La préparation de programmes de travail annuels se heurte toutefois au problème majeur de savoir si le processus en question relève d'une approche purement «ascendante » dans laquelle le BG se contente de regrouper les questions transmises par les ministères, ou si le BG a le pouvoir et les moyens d'exercer un jugement dans la compilation du programme sur la base des éléments fournis. En d'autres termes, le Bureau du Gouvernement vérifie, par exemple, que les plans soumis par les ministères tiennent suffisamment compte des priorités du gouvernement et peut demander à un ministère des éléments supplémentaires en cas d'omission d'éléments nécessaires à la réalisation des priorités stratégiques. Inversement, il peut stipuler à un ministère qu'il a inclus trop d'éléments, certains d'entre eux ne pouvant être traités par le gouvernement ou le parlement par manque de temps. De cette manière, la préparation du programme de travail devient un

3 L'évaluation la plus récente de Sigma sur la coordination des politiques dans les PECO remonte à 2000 ; des évaluations ont été réalisées en 2003 /4 auprès des pays/entités des Balkans occidentaux. 
processus interactif entre les ministères, lesquels favorisent des priorités sectorielles spécifiques, et le BG, auquel il incombe de prendre en considération les questions intersectorielles et celles touchant le gouvernement dans son ensemble. Inutile de dire que la décision finale concernant le programme de travail, y compris le règlement de tout conflit, incombe au CdM.

\subsection{Coordination du contenu politique des propositions pour le CdM (préparation dans les ministères, activités du BG)}

En règle générale, les systèmes d'élaboration des politiques et des projets de lois assignent à chaque ministre les tâches suivantes :

- Identifier les problèmes exigeant une réponse du gouvernement ;

- $\quad$ Initier l'élaboration de solutions politiques ;

- Consulter les ministres et les groupes extérieurs intéressés ;

- $\quad$ Analyser les conséquences des solutions proposées ; et

- Présenter une proposition de solution (ou de quelques options de solution) pour une décision collective par le CdM.

Dans l'exercice de ces tâches, le ministre est assisté de son ministère. En cas de questions complexes, le ministre peut chercher le soutien additionnel d'experts extérieurs ou d'un groupe de travail spécial (y compris des groupes de travail interministériels). L'impulsion nécessaire pour initier l'élaboration d'une politique peut découler des priorités du gouvernement, du travail d'analyse du ministère concerné, d'obligations internationales ou encore de pressions extérieures en provenance des entreprises, des groupes d'intérêt ou des citoyens. Indépendamment de l'impulsion initiale, les premières étapes de ce processus incombent au ministre, appuyé par son ministère.

D'autre part, dans la mesure où les décisions politiques relèvent de la responsabilité collective du gouvernement, il est nécessaire de garantir au CdM dans son ensemble que les ministères ont préparé des propositions spécifiques conformément à un processus approprié, que ces propositions sont adaptées aux plans, au calendrier et aux priorités du gouvernement, et qu'elles ne viennent pas contredire d'autres décisions. C'est là tout l'enjeu de la coordination des politiques, une responsabilité tout naturellement dévolue au BG en sa qualité d'organisme central au service du CdM.

La coordination des politiques constitue l'outil central du BG dans sa mission de servir la responsabilité collective du CdM d'élaborer et de mettre en application des politiques cohérentes. Le BG doit plus particulièrement être en mesure de garantir la cohérence, la logique et la bonne coordination des questions soumises au gouvernement et à ses comités ministériels.

À cette fin, le BG doit exercer les activités suivantes :

- $\quad$ S'assurer que les questions sectorielles et intersectorielles ont bien été prises en compte et analysées sous tous les aspects ;

- S'assurer que l'analyse et les pièces justificatives (budgétaires, économiques, sociales, environnementales, etc.) sous-tendant les propositions des ministères sont de très bonne qualité ;

- $\quad$ S'assurer que les propositions des ministres sont conformes aux priorités stratégiques et budgétaires du gouvernement ;

- $\quad$ S'assurer que les désaccords entre ministères sur les documents fournis au gouvernement sont résolus ou atténués avant les sessions du CdM ; et

- $\quad$ S'assurer que le Premier ministre et les présidents des comités ministériels sont bien informés des questions soumises aux comités et au CdM pour décision.

\subsubsection{Préparation des documents dans les ministères : rôle du BG dans l'établissement} de procédures d'analyse des politiques

La préparation et l'élaboration de propositions pour le CdM relève de la responsabilité des ministres et de leurs ministères dans tous les pays couverts ici. Le processus d'élaboration des politiques et des lois en luimême dépasse la portée du présent document. Néanmoins, il est important de noter que dans de nombreux 
pays, le BG assure la mission centrale d'encadrer le processus de préparation, notamment en définissant les règles de soumission au CdM. Pour simplifier, si le BG exige de soumettre au CdM certains types d'informations et d'analyses, le ministère à l'origine de la proposition devra normalement conduire ces analyses dans son processus de préparation et les inclure dans sa présentation.

Pour cette raison, il est courant de voir les Bureaux de gouvernement de nombreux pays membres de l'OCDE (par exemple, le Canada, le Royaume-Uni et le Danemark) préparer des instructions manuelles à l'attention des ministères sur le type spécifique d'informations requises par les ministres pour les débats du CdM. Dans la mesure où l'analyse et la recherche fournies à l'appui d'une proposition pour la justifier sont préparées par chaque ministre et son ministère, les instructions générales ont pour but de s'assurer que les questions les plus importantes seront traitées et mises en lumière dans une brève synthèse (généralement de cinq pages environ) dont le format de présentation est fixé. Cette synthèse, si elle est bien préparée, permet d'axer les débats du CdM sur les questions importantes, plutôt que sur les détails techniques et rédactionnels (mieux traités s'ils sont laissés à des experts). En outre, elle permet de discipliner le processus de préparation, est utile aux discussions internes au sein du ministère, et constitue une bonne base pour le BG, en ce sens qu'elle examine la proposition à l'étude dans un contexte politique élargi.

Les éléments spécifiques contenus dans cette brève synthèse remise aux ministres varient quelque peu d'un pays à l'autre ainsi que sur le temps, au même titre que le format de présentation. Il est important de noter qu'il est de la responsabilité du BG de s'assurer que les ministres reçoivent les informations dont ils ont besoin pour prendre des décisions éclairées. Alors que de telles informations doivent être fournies par le ministère à l'origine de la proposition, il incombe en dernier ressort au BG de veiller à leur qualité et à leur exhaustivité. Si elle est bien préparée, cette brève synthèse offre souvent un excellent aperçu de la proposition aux ministres qui se préparent pour la session du $\mathrm{CdM}$, et sert souvent de référence principale à leurs débats au cours de la session elle-même. 
Les principales questions devant généralement être soumises au CdM sont répertoriées dans les deux exemples exposés ci-après.

\section{Exemple général de format pour la brève synthèse soumise au $\mathrm{CdM}$}

\section{1. $\quad \operatorname{Décision}(\mathbf{s})$ requise(s)}

Cette section indique au CdM, en une ou deux phrases, la ou les questions que l'auteur de la proposition lui demande d'étudier et d'approuver

\section{Options considérées}

Cette section explique brièvement les options qui ont été considérées par l'auteur de la proposition. Des informations descriptives or contextuelles critiques devraient être fournies pour chaque option, de préférence sous forme de commentaires en une/deux phrases Ces commentaires doivent englober les principales implications de la proposition, ainsi que les pour et les contre.

\section{Principal résultat des consultations}

Cette section doit identifier les ministères, les ONG et tout autre groupe cible que la proposition est susceptible de considérablemen affecter, et mettre en lumière les parties consultées et dont les principales préoccupations n'ont pas été résolues. Il est important que ces opinions se reflètent de manière franche et exacte dans la synthèse.

\section{Option recommandée et raisonnement}

Cette section doit indiquer l'option recommandée et expliquer le raisonnement ayant dicté le choix de l'option recommandée par rapport aux autres solutions. Dans la mesure du possible, il conviendra de corréler la proposition avec les priorités stratégiques et les autres engagements du gouvernement et les décisions du CdM. Toutes considérations fondées sur la rentabilité et les attitudes publiques sont également une source d'informations utiles à inclure dans cette section.

\section{Coût de l'option recommandée}

Sur la base d'une analyse financière complète, cette section doit indiquer le coût prévu de l'option recommandée et, le cas échéant, suggérer la source de financement (par exemple, fonds propres du ministère, budget de l'année suivante, réserves, etc.). Dan\$ certains cas (par exemple, dans l'ancienne République yougoslave de Macédoine), cette section doit être accompagnée d'une évaluation budgétaire complète des répercussions.

\section{Répercussions escomptées}

Cette section récapitule brièvement l'impact que cette décision peut avoir, par exemple, sur le public, les groupes cibles, les contribuables, l'économie, l'emploi, l'environnement. Cette synthèse doit s'appuyer sur l'analyse et sur toute recherche quantitative contenue dans le dossier soumis au CdM.

\section{Harmonisation avec la législation européenne}

Cette section doit brièvement expliquer l'importance, quelle qu'elle soit, des documents proposés en matière d'harmonisation avec la législation européenne. Dans le cas de projets de lois, elle doit indiquer si les documents ont été étudiés et certifiés par l'organisme compétent responsable de l'harmonisation juridique.

\section{Messages de communication}

Cette section doit suggérer au CdM un petit nombre de messages de communication stratégique à utiliser lors de l'annonce de cette décision et/ou au moment d'expliquer pourquoi le $\mathrm{CdM}$ a décidé de l'adopter. Cet élément se révèle particulièrement important dans les cas où une décision risque d'être impopulaire auprès du grand public ou auprès de groupes publics spécifiques, ou de ne pas être respectée du public. 
Ministre de la Santé

\section{MÉMORANDUM DE CONFIANCE ADRESSE AU \\ GOUVERNEMENT AU CONSEIL

Intitulé exposant de manière concise la substance du mémorandum

\section{Contexte}

Le but de ce mémorandum est d'obtenir l'approbation du gouvernement pour un nouveau plan de remboursement des médicaments sur ordonnance. Le 22 octobre 1997, la commission désignée pour examiner l'introduction d'un tel plan a présenté ses recommandations..

\section{Solutions alternatives}

J'ai considéré diverses façons de résoudre la question. L'une d'elles peut consister à....

3. Arguments pour et contre la proposition Arguments pour Arguments contre

\section{Mes recommandations}

Après avoir étudié les diverses options, j'ai décidé de recommander l'alternative 2, pour les motifs suivants :

\section{Soumission pour commentaires}

Le mémorandum du gouvernement a été soumis au Ministre des finances et au Ministre de l'environnement. Le Ministre des finances n'a fait aucune observation. Le Ministre de l'environnement a fait le commentaire suivant :

$»($ Conclusion alternative, si appropriée $) \ll$

\section{Conclusion}

1. Il conviendrait d'introduire un plan en vertu duquel...

2. Le coût de ce plan doit être couvert dans le cadre du budget existant du ministère de la Santé... 


\subsubsection{Examen des politiques au sein du BG : coordination et résolution des conflits}

Une fois qu'une proposition destinée au CdM est soumise au BG, il est important d'en examiner le contenu pour s'assurer qu'elle est prête à être débattue et décidée en session ou au sein des comités ministériels. La plupart des BG dans les pays de l'OCDE énoncent parmi leurs principales fonctions la coordination des politiques ainsi que la résolution des litiges ; certains indiquent mêmes ces deux éléments comme leur unique fonction. En Allemagne, par exemple, une des trois fonctions énumérées dans les prérogatives du BG est « de fournir au Chancelier les informations et les ressources en matière de coordination nécessaires pour la conduite de la politique générale du gouvernement ». L'Irlande indique comme principale fonction du BG le fait « de fournir des conseils politiques et une assistance administrative au Taoiseach [Premier ministre] ». En Nouvelle-Zélande, la principale fonction du BG est « de favoriser l'intérêt collectif du gouvernement et la coordination efficace du processus d'élaboration des politiques». En Turquie, une des fonctions du BG consiste à «assurer la coordination entre les ministères, superviser la gestion du programme général de la politique du gouvernement ».

La situation est relativement différente dans les PECO et les pays des Balkans occidentaux où, comme les évaluations de Sigma l'indiquent, les BG sont principalement affaiblis par le manque de moyens politiques. Évidemment, quelques signes encourageants suggèrent que cette situation est en train de changer. Au nombre des exemples de cette évolution figure la Hongrie où, depuis 1998, un Bureau de gouvernement restructuré s'est vu confier un rôle plus influent dans la mise en application des objectifs du gouvernement ainsi que dans la mise en place d'une coordination substantielle. Un groupe d'experts, appelé «Referatura », chapeaute les ministères pour assurer une coordination horizontale. La Lettonie a doté son BG d'un Département de coordination des politiques, et l'ancienne République yougoslave de Macédoine est également près de finaliser l'instauration d'un département similaire. D'autres pays, comme par exemple la Bulgarie et la Slovaquie, possèdent des BG déjà dotés de certains moyens politiques, mais doivent encore en renforcer les compétences et l'expertise.

La coordination des politiques est un processus complexe et parfois évasif. On ne s'attend pas à ce que le personnel assigné à l'analyse des politiques du BG possède une connaissance approfondie d'un sujet spécifique traité dans un ministère, ni à ce qu'il tente de se substituer aux experts des ministères dans l'élaboration des politiques. En revanche, les membres de ce personnel tendent à se poser en « généralistes de secteur ", c.-à-d., en économistes ou experts en matière de politique sociale ou extérieure, possédant une vision globale du secteur dans son ensemble. Une fois qu'ils étudient une proposition, ils ont généralement à leur disposition quatre outils de coordination :

- Perspective élargie : Ils peuvent employer leurs connaissances des priorités du gouvernement et du secteur dans son ensemble pour indiquer au personnel ministériel les points de la proposition susceptibles d'exiger un ajustement.

- Garants du processus : Ils peuvent aviser et rappeler aux ministères les règles de préparation des propositions à soumettre au CdM ainsi que l'importance de procéder à des analyses de grande qualité et d'ouvrir les consultations, en mettant l'accent sur les points du processus susceptibles d'être mieux gérés.

- Résolution des conflits : Ils peuvent convoquer et présider des réunions interministérielles de hauts fonctionnaires (formelles ou informelles) afin de résoudre les conflits et d'élaborer des solutions.

- Informer les supérieurs hiérarchiques : En cas d'impossibilité à résoudre les questions importantes, ils peuvent instruire le Premier ministre, les présidents des comités ministériels, ou le Secrétaire Général sur les problèmes d'une proposition, de sorte que les questions en suspens puissent être reprises à un niveau hiérarchique supérieur. Ils peuvent proposer au Secrétaire Général de reprendre les questions non résolues lors de la réunion hebdomadaire des secrétaires d'État et suggérer au Premier ministre (par l'intermédiaire du Secrétaire Général) la façon de gérer ladite question en session du CdM.

Une implication anticipée des ministères dans le processus d'élaboration des politiques est essentielle pour garantir une utilisation efficace des outils susmentionnés. En règle générale, le personnel en charge des analyses politiques au sein du BG est assigné au suivi d'un ministère, d'un certain nombre de ministères ou d'un secteur entier (économique, social, étranger et défense). Lorsqu'une initiative significative de politique est élaborée dans l'un des ministères assignés à cette tâche, le personnel du BG est censé en être informé par l'entremise de réseaux formels ou informels. Il peut ainsi être en mesure d'assister aux réunions des groupes 
de travail préparatoires ou de discuter des questions de manière informelle avec les personnes ayant préparé les concepts ou les premières versions provisoires. Il peut proposer de convoquer des réunions interministérielles anticipées d'experts pour discuter des questions avant qu'elles ne soient décidées, ou soumettre des idées supplémentaires au sujet des sources d'informations et de l'expérience internationale.

\section{Pour un tableau récapitulatif de la participation du BG à l'examen du contenu des politiques, prière de se reporter au Tableau 3, ci-dessus.}

Une description du mode de fonctionnement de la Referatura en Hongrie permet d'illustrer l'emploi de certains de ces outils. Les experts sont impliqués dès les premières étapes de la préparation des documents des ministères, ainsi ils sont bien informés de leur contenu avant que les documents ne soient transmis au BG. Le chef du bureau responsable au sein de la Referatura prépare une note sur chaque document, récapitulant le contexte, la description de la proposition, le résultat des consultations interministérielles, les points non résolus, les questions que le $\mathrm{BG}$ juge problématiques, et les propositions pour résoudre de tels problèmes. Cette note est remise au Premier ministre, au ministre responsable vis-à-vis du BG, au chef de la Referatura et au porte-parole du gouvernement.

Pour résoudre les conflits interministériels sur des aspects politiques avant décision définitive du CdM, les réunions des hauts fonctionnaires organisées et/ou menées par le BG sont un outil particulièrement utilisé. Ces réunions peuvent être très formelles, comme en France (arbitrage), ou informelles, comme au Canada. Elles peuvent être en comité restreint, et ne requérir la participation que de deux ministères en cas de problème sérieux (par exemple en cas de conflit fondamental entre l'objectif du Ministère des transports et celui du Ministère d'environnement). Elles peuvent au contraire réunir tous les ministères et même les agences et les bureaux régionaux concernés. Les membres du personnel du BG qui convoquent et président de telles réunions ont l'avantage de pouvoir agir en tant qu'«intermédiaires neutres » puisqu'ils ne représentent les intérêts d'aucun ministère, mais uniquement la mission collective du gouvernement.

En guise d'étape finale de coordination avant que les questions ne soient étudiées par les ministres (en comités ou en session plénière du CdM), de nombreux pays (notamment les PECO, semble-t-il) jugent utile de tenir une réunion des secrétaires d'État de tous les ministères, présidée par le Secrétaire Général. On observe de telles réunions, par exemple, en Hongrie, en Lituanie, en Lettonie, en ancienne République yougoslave de Macédoine, en Estonie, en Espagne, en Norvège, en Finlande et en Allemagne. Pour cette réunion, le Secrétaire Général doit être bien informé par le personnel d'analyse des politiques du BG, de manière à pouvoir se concentrer sur les questions non résolues et peut-être en résoudre certaines avant de soumettre les questions aux ministres pour décision. Là encore, une implication anticipée et continue du personnel du BG sur les questions soulevées et avec les ministères auxquels il est affecté augmentera la qualité des informations qu'il transmet au Secrétaire Général.

Pour finir, beaucoup de pays ont développé un système de comités ministériels qui se réunissent pour discuter des questions de manière approfondie et pour résoudre définitivement les conflits avant la session plénière du CdM. Ces comités, parfois désignés sous le nom de «comités de filtrage », rassemblent les ministres (et parfois les fonctionnaires qui les assistent), habituellement dans le cadre d'un regroupement sectoriel élargi, par exemple comité de politique économique, comité des affaires sociales, ou comité pour l'intégration européenne. Ces comités peuvent être présidés par le Premier ministre, un vice premier ministre, ou un ministre senior dans le domaine spécifique. L'examen réalisé par ces comités peut être requis pour toutes les questions à transmettre au $\mathrm{CdM}$, ou des comités peuvent être convoqués pour discuter de questions spécifiques revêtant une importance politique ou stratégique. Dans la plupart des cas, le travail de ces comités est soutenu par le BG. 
Tableau 5. Réunions de coordination préalables à la réunion du $\mathrm{CdM}$

\begin{tabular}{|c|c|c|}
\hline Pays membres de l'OCDE & $\begin{array}{c}\text { Les questions sont-elles discutées par les } \\
\text { comités ministériels préalablement à la } \\
\text { décision du CdM? }\end{array}$ & $\begin{array}{l}\text { Les hauts fonctionnaires ministériels se } \\
\text { réunissent-ils chaque semaine pour } \\
\text { discuter et finaliser les questions à } \\
\text { l'ordre du iour? }\end{array}$ \\
\hline Australie & Oui, c'est une condition & Non \\
\hline Autriche & Oui, c'est une condition & Oui, chaque semaine \\
\hline France & Oui, c'est une condition & $\begin{array}{c}\text { Non (mais large recours aux réunions } \\
\text { d'arbitrage) }\end{array}$ \\
\hline Allemagne & Oui, c'est une condition & Oui, chaque semaine \\
\hline Islande & Seules les questions importantes sont & Non \\
\hline Irlande & $\begin{array}{l}\text { Seules les questions importantes sont } \\
\text { discutées par les comités }\end{array}$ & Non \\
\hline Pays-Bas & Oui, c'est une condition & Non \\
\hline Norvège & Non & Oui, chaque semaine \\
\hline Espagne & Oui, c'est une condition & Oui, chaque semaine \\
\hline Suède & $\begin{array}{c}\text { Non, il n'y aucun comité ministériel. Les } \\
\text { questions sont discutées entre les ministres } \\
\text { lors de délibérations générales }\end{array}$ & Non \\
\hline
\end{tabular}

\begin{tabular}{|c|c|c|}
\hline PECO & $\begin{array}{c}\text { Les questions sont-elles discutées par les } \\
\text { comités ministériels préalablement à la } \\
\text { décision du CdM? }\end{array}$ & $\begin{array}{l}\text { Les hauts fonctionnaires ministériels se } \\
\text { réunissent-ils chaque semaine pour } \\
\text { discuter et finaliser les questions à }\end{array}$ \\
\hline Bulgarie & Seules les questions importantes sont & Oui, chaque semaine \\
\hline République Tchèque & Seules les questions importantes sont & Oui, chaque semaine \\
\hline Estonie & Non & Oui, chaque semaine \\
\hline Hongrie & Oui, c'est une condition & Oui, chaque semaine \\
\hline Lettonie & Oui, c'est une condition & Oui, chaque semaine \\
\hline Lituanie & Seules les questions importantes sont & Oui, chaque semaine \\
\hline Pologne & Oui, c'est une condition & Oui, chaque semaine \\
\hline Slovaquie & Seules les questions importantes sont & Non \\
\hline Slovénie & Oui, c'est une condition & Oui, chaque semaine \\
\hline
\end{tabular}

\begin{tabular}{|l|c|c|}
\hline \multicolumn{1}{|c|}{ Pays des Balkans occidentaux } & $\begin{array}{c}\text { Les questions sont-elles discutées par les } \\
\text { comités ministériels préalablement à la } \\
\text { décision du CdM ? }\end{array}$ & $\begin{array}{c}\text { Les hauts fonctionnaires ministériels se } \\
\text { réunissent-ils chaque semaine pour } \\
\text { discuter et finaliser les questions à }\end{array}$ \\
\hline Albanie & Seules les questions importantes sont & Parfois \\
\hline Bosnie-Herzégovine (au niveau de l'état) & Oui, c'est une condition & Non \\
\hline Bosnie-Herzégovine (Fédération) & Non & Non \\
\hline Croatie & Oui, c'est une condition & Oui \\
\hline Ancienne République yougoslave de & Oui, c'est une condition & Non \\
\hline Serbie et Monténégro (Fédération) & Oui, c'est une condition & Oui, chaque semaine \\
\hline Serbie-Monténégro / Monténégro & Oui, c'est une condition & Oui, chaque semaine \\
\hline Serbie-Monténégro / Kosovo & Seules les questions importantes sont & \\
\hline
\end{tabular}


Un outil très utile pour garantir que les efforts du BG en matière de coordination des politiques influencent l'issue des débats du $\mathrm{CdM}$ consiste à préparer une brève note à l'intention du Président du $\mathrm{CdM}$ (généralement le Premier ministre). De telles notes permettent au BG d'aviser le Président des questions en suspens ou des conflits non résolus entre les ministères qui pourraient requérir une attention particulière en session. Le fait d'informer le Président avant les sessions confère également au BG un certain statut dans le système, ainsi qu'un certain pouvoir informel de requérir de meilleures informations et de résoudre les conflits. Si les ministères savent que le Président peut être avisé dans cette note d'instruction que les questions ne sont pas prêtes pour le $\mathrm{CdM}$, ils seront incités à fournir des efforts supplémentaires pour résoudre les problèmes et éviter un dossier «négatif ».

Tableau 6. Conseils du Bureau du Gouvernement sur les politiques

\begin{tabular}{|c|c|c|c|}
\hline ys membres de l'OCDE & $\begin{array}{c}\text { Le BG prépare-t-il une note à } \\
\text { l'attention du Président du CdM } \\
\text { sur les questions figurant à l'ordre } \\
\text { du jour des réunions du CdM? }\end{array}$ & $\begin{array}{l}\text { La note adressée au Président } \\
\text { comprend-elle des } \\
\text { recommandations sur le } \\
\text { traitement à réserver aux } \\
\text { auestions lors de la réunion? }\end{array}$ & $\begin{array}{c}\text { Les notes adressées au Président } \\
\text { sont-elles diffusées aux autres } \\
\text { membres du CdM? }\end{array}$ \\
\hline Australie & Oui, pour chaque question & Oui & Non, uniquement au Président \\
\hline Autriche & Non & Non & Aucune note n'est préparée \\
\hline France & Oui, pour chaque question & Oui (par le CPM) & $\begin{array}{l}\text { Non, seulement au Président et au } \\
\text { Porte-parole }\end{array}$ \\
\hline Allemagne & Oui, pour chaque question & Oui & Non, uniquement au Président \\
\hline Islande & Non & Aucune note n'est préparée & Aucune note n'est préparée \\
\hline Irlande & Oui, pour chaque question & Parfois & Non, uniquement au Président \\
\hline Pays-Bas & Oui, pour chaque question & Oui & Non, uniquement au Président \\
\hline Norvège & Oui, pour chaque question & Oui & Non, uniquement au Président \\
\hline Espagne & Seulement pour certaines questions & Parfois & Non, uniquement au Président \\
\hline Suède & Seulement pour certaines questions & Oui & Non, uniquement au Président \\
\hline
\end{tabular}

\begin{tabular}{|l|c|c|c|}
\hline PECO & $\begin{array}{c}\text { Le BG prépare-t-il une note à } \\
\text { l'attention du Président du CdM } \\
\text { sur les questions figurant à l'ordre } \\
\text { du jour des réunions du CdM ? }\end{array}$ & $\begin{array}{c}\text { La note adressée au Président } \\
\text { comprend-elle des } \\
\text { recommandations sur le } \\
\text { traitement à réserver aux }\end{array}$ & $\begin{array}{c}\text { Les notes adressées au Président } \\
\text { sont-elles diffusées aux autres } \\
\text { membres du CdM ? }\end{array}$ \\
\hline Bulgarie & Oui, pour chaque question & Oui & Oui, à tous les membres \\
\hline République Tchèque & Oui, pour chaque question & Oui & Non, uniquement au Président \\
\hline Estonie & Oui, pour chaque question & Parfois & Non, uniquement au Président \\
\hline Hongrie & Oui, pour chaque question & Oui & Non, uniquement au Président \\
\hline Lettonie & Seulement pour certaines questions & Oui & Non, uniquement au Président \\
\hline Lituanie & Oui, pour chaque question & Oui & Oui, à tous les membres \\
\hline Pologne & Oui, pour chaque question & Oui & Non, uniquement au Président \\
\hline Slovaquie & Seulement pour certaines questions & Parfois & Non, uniquement au Président \\
\hline Slovénie & Oui, pour chaque question & Oui & Oui, à tous les membres \\
\hline
\end{tabular}

\begin{tabular}{|l|c|c|c|}
\hline $\begin{array}{c}\text { Pays des Balkans } \\
\text { occidentaux }\end{array}$ & $\begin{array}{c}\text { Le BG prépare-t-il une note à } \\
\text { l'attention du Président du CdM } \\
\text { sur les questions figurant à l'ordre } \\
\text { du jour des réunions du CdM ? }\end{array}$ & $\begin{array}{c}\text { La note adressée au Président } \\
\text { comprend-elle des } \\
\text { recommandations sur le } \\
\text { traitement à réserver aux }\end{array}$ & $\begin{array}{c}\text { Les notes adressées au Président } \\
\text { sont-elles diffusées aux autres } \\
\text { membres du CdM ? }\end{array}$ \\
\hline Albanie & Oui, pour chacune d'elles & Parfois & Non, uniquement au Président \\
\hline Bosnie-Herzégovine (au & Non & Non & Aucune note n'est préparée \\
\hline Bosnie-Herzégovine & Non & Non & Aucune note n'est préparée \\
\hline Croatie & Non & Aucune note n'est préparée & Aucune note n'est préparée \\
\hline Ancienne République & Non & Non & Aucune note n'est préparée \\
\hline Serbie et Monténégro & Oui, pour chaque question & Non & Non, uniquement au Président \\
\hline Serbie-Monténégro / & Oui, pour chaque question & Oui & Oui, à tous les membres \\
\hline $\begin{array}{l}\text { Serbie-Monténégro / } \\
\text { Kosovo }\end{array}$ & Seulement pour certaines questions & Parfois & $\begin{array}{c}\text { Seulement aux ministres } \\
\text { directement concernés }\end{array}$ \\
\hline
\end{tabular}


En matière de préparation des dossiers politiques, les réponses au questionnaire laissent entrevoir que les PECO ont considérablement progressé ces dernières années, depuis les dernières évaluations de Sigma réalisées en 2000 sur l'élaboration des politiques. Sauf dérive du système d'auto-notation, on observe un rôle croissant du BG particulièrement bienvenu dans les sujets liés aux politiques. À l'instar des nombreuses questions abordées dans le présent rapport, les pays des Balkans occidentaux semblent souffrir également de retards dans ce domaine.

\subsection{Coordination des messages de communication}

Au cours des dernières décennies, les rapports entre politique et communications n'ont cessé de prendre de l'ampleur, au même titre que les rapports entre les hommes politiques et les médias. Un phénomène ayant pour conséquence d'entrecroiser le processus d'élaboration des politiques et les communications du gouvernement. Au nombre des raisons expliquant le lien étroit entre l'élaboration des politiques et les communications figurent notamment les éléments suivants :

- Les démocraties modernes se caractérisent de plus en plus par un besoin fondamental de transparence, qui impose le devoir aux gouvernements d'expliquer au grand public ses actions et les raisons qui les sous-tendent. L'accès aux informations sur les politiques et les décisions gouvernementales est désormais considéré comme un droit fondamental des citoyens.

- $\quad$ Le succès des actions gouvernementales dépend en dernier ressort de la tolérance et de l'acceptation du public : si une loi ou un impôt sont perçus comme injustes, il y a de grandes chances qu'ils soient enfreints ou contournés. Le fait d'expliquer le raisonnement qui sous-tend les décisions gouvernementales augmente la probabilité de voir aboutir avec succès les actions du gouvernement.

- $\quad$ Si le gouvernement omet d'expliquer les raisons de ses actions, il entrouvre la voie aux spéculations selon lesquelles ses motifs ne seraient pas licites. Si la presse pose des questions auxquelles le gouvernement ne répond pas correctement, ou si les réponses des ministres sont contradictoires, beaucoup de personnes en tireront des conclusions défavorables.

- De nombreuses actions gouvernementales créent à la fois des «gagnants » et des «perdants». Les perdants d'une nouvelle politique gouvernementale - que ce soit un système fiscal reformé ou la décision de construire une nouvelle route - risquent de se faire davantage entendre que les gagnants, et la presse est plus susceptible de se faire l'écho de ces critiques. Afin de soutenir sa politique, un gouvernement a intérêt à dire toute la vérité.

- Les gouvernements sont très sensibles à leur image publique pendant toute la durée de leur mandat, et particulièrement à l'approche d'élections. Ils ont tout intérêt à influencer la manière de communiquer les nouveaux développements ainsi que le contenu et le moment des annonces. Si chaque ministre est totalement libre de communiquer ses annonces à la presse, la gestion collective du gouvernement et de ses politiques risque d'apparaître contradictoire.

L'accroissement de la demande publique en informations a également augmenté l'intérêt des gouvernements à contrôler et à coordonner leurs messages afin de renforcer la popularité et l'acceptation de leurs politiques. Ce contrôle et cette coordination par le gouvernement empêchent également les ministères de communiquer des messages différents et contradictoires. En conséquence, beaucoup de gouvernements développent une approche collective de leurs communications, en conférant généralement au Premier ministre la responsabilité globale de s'assurer que le public est informé des travaux du gouvernement. D'autre part, les ministres sont responsables, en consultation avec le Premier ministre, de veiller à ce que le public soit au courant des développements dans leur propre domaine de compétences. La gestion et la coordination quotidiennes du fonctionnement des communications sont souvent dévolues au BG et au CPM, directement entre les mains du porte-parole du Gouvernement ou du Chef du bureau presse du Gouvernement/Premier ministre.

Le BG établit alors des mécanismes pour s'assurer, par exemple, que les informations fournies par un ministère sont cohérentes avec les informations émises par les autres ministères et par le porte-parole du gouvernement, et que les initiatives sont synchronisées et les annonces programmées pour en accroître l'impact. Au nombre des mécanismes de coordination figurent : l'obligation d'indiquer les messages de communication dans le cadre de la note de synthèse soumise au CdM (voir l'exemple ci-dessus) ; réunions hebdomadaires des conseillers en communication des ministres, présidées par le porte-parole du Gouvernement ; point hebdomadaire sur les communications lors de la réunion du CdM ; et planification stratégique des communications. 
Dans de nombreux pays, les moyens de coordination des communications du gouvernement sont dévolus au BG et/ou au CPM. En Slovénie, par exemple, le Bureau des relations publiques et des médias est en partie un service, en partie un mécanisme de coordination. Il fournit à l'ensemble du gouvernement un soutien complet en matière de relations publiques et d'informations, et divulgue les informations au public, dans le pays et à l'étranger, y compris en matière d'IE. Son directeur est le porte-parole officiel du Gouvernement. Ce bureau coordonne également les relations publiques de travail des ministères, dans le cadre d'un réseau bien établi de représentants ministériels. En Autriche, l'un des cinq départements de la Chancellerie fédérale est appelé «l'Unité fédérale presse et information », et la Chancellerie allemande comprend un Bureau gouvernemental presse et informations, largement doté en effectifs, rattaché à un secrétaire d'état.

Les communications gouvernementales relèvent presque toujours de l'exercice d'équilibriste entre information et propagande. Dans une démocratie, il convient d'opérer une distinction complexe entre aviser et influencer le public. Pour cette raison, la coordination et la gestion des informations et des communications gouvernementales sont souvent dévolues à la division politique du BG (CPM), plutôt qu'à sa division administrative. Cependant, dans de nombreux pays, ces prérogatives relèvent à la fois du BG et CPM, le premier étant responsable des «informations» et le second des «communications ». Cette distinction souligne l'obligation collective du gouvernement de tenir le public informé ainsi que son devoir politique d'expliquer, de justifier, et même de « vendre » ses politiques et ses lois au public.

\subsection{Coordination du suivi de l'exécution gouvernementale}

L'application des décisions gouvernementales et le suivi des résultats relèvent de la responsabilité fondamentale de chaque ministre concerné. Cependant, le gouvernement dans son ensemble, et plus particulièrement le Premier ministre personnellement, sont soumis à un devoir (constitutionnel, juridique ou par convention) de performance aussi bien individuelle que collective. Dans les informations communiquées dans l'établissement des profils PUMA susmentionnés, les Pays-Bas résument succinctement cet aspect : «L'application des décisions du Conseil des Ministres relève de la responsabilité des ministres concernés. Le programme du gouvernement et l'avancée des propositions de la politique projetée sont régulièrement examinés par le Conseil des Ministres. Le rapport d'avancement est compilé par le Secrétaire du Conseil des Ministres [chef du BG]. ». La plupart des pays de l'OCDE et de nombreux PECO et pays des Balkans occidentaux adoptent une approche semblable, et la majorité des BG disposent au moins de ressources limitées pour assurer le suivi des résultats.

Dans ce domaine, le problème le plus courant auquel sont confrontés les PECO et les pays des Balkans occidentaux est que ce suivi est souvent axé de manière quasi-exclusive sur ses aspects formels ; en d'autres termes, les aspects tels que les tâches assignées et des dates d'exécution font l'objet d'un suivi (souvent via un système informatisé), mais les impacts et les effets réels sur la société et l'économie ne sont pas contrôlés. Cette tendance est à rapprocher de la définition excessivement juridique de l'« application » qui est assimilée au passage d'une loi et/ou d'une loi dérivée (réglementation). Dans ce contexte, une décision est jugée avoir été appliquée dès lors que la loi est votée, et un ministre est réputé avoir appliqué une décision du CdM une fois la législation dérivée requise approuvée.

S'il ne fait aucun doute que le passage d'actes juridiques est une étape nécessaire en vue d'une application intégrale, il n'est sûrement pas raisonnable de dire qu'un budget, un impôt ou un programme scolaire « est appliqué » lorsque la loi et les réglementations de leur exécution sont votées. L'application implique plutôt certaines étapes concrètes, par exemple un nouveau programme scolaire est appliqué lorsque tous (ou la quasi-totalité) les professeurs et écoles l'utilisent. Un des enjeux de la réforme de l'administration publique dans les PECO et les pays des Balkans occidentaux est d'élargir les concepts d'application et de suivi au-delà de leurs définitions purement formelles et juridiques. Le BG peut y contribuer en étendant la définition de ce qui constitue la performance collective, ainsi que ses activités de suivi pour se concentrer sur l'application réelle et concrète des politiques et de la législation.

\subsection{Coordination des échanges avec les autres instances de l'État (Président, Parlement)}

Le maintien de la cohérence des politiques doit souvent se poursuivre au-delà de la décision du CdM. Dans tous les pays couverts ici, le parlement doit passer toute la législation primaire, et peut tenter d'amender la législation du gouvernement dans le processus. En outre, le parlement initie également des lois, lesquelles peuvent bien s'harmoniser ou non avec les propres priorités et politiques du gouvernement. Ce point se vérifie plus particulièrement dans les PECO, où les parlements tendent à initier un grand nombre de lois, et où, en 
outre, il y existe souvent des coalitions difficiles et instables, si bien que le gouvernement peut avoir des problèmes à contrôler son agenda politique. Il est donc nécessaire de coordonner les échanges avec le parlement pour s'assurer que les ministres sont prêts à discuter et à défendre les lois au sein de leurs portefeuilles, et à présenter une position commune si nécessaire.

Autre intermédiaire de poids pour le gouvernement : le Président (et son bureau). C'est généralement le cas dans les systèmes semi-présidentiels, où l'on observe généralement des domaines communs et corrélés de responsabilité entre le Président et le Premier ministre. Mais, même dans les régimes purement parlementaires, le chef d'état influence la politique, du moins ponctuellement, et dans tous les cas, vise habituellement les projets législatifs en lois, mais pas toujours de manière automatique.

Pour ces raisons, il est assez courant de confier au BG la responsabilité de gérer et de coordonner les échanges avec le parlement pour le compte du gouvernement, notamment en matière de planification et de programmation de la législation gouvernementale. Par exemple, en Italie, en Belgique, en France et en Allemagne, le BG comprend une unité chargée de gérer les questions liées au parlement. Dans les PECO, cette fonction revêt souvent une portée assez significative. En Lituanie, cette mission incombe à un haut responsable au sein du BG. En Pologne, le BG compte parmi ses principales missions l'obligation de « coordonner la coopération entre le Conseil des Ministres et le Premier ministre et le Sejm (Parlement), le Sénat et le Président de la République. Au nombre des tâches assignées à de telles unités, figurent le suivi du passage des projets de lois du gouvernement, l'organisation et la programmation des interventions des membres du gouvernement au parlement, la soumission d'informations dans la préparation de «l'heure gouvernementale » ou de la session des questions au parlement, le suivi des réponses écrites aux questions des députés, et la transmission d'informations et de conseils généraux sur les échanges avec le parlement et les différents députés. ».

Dans les régimes parlementaires, les unités chargées des échanges avec le chef de l'état sont plus rares par rapport aux unités de coordination avec le parlement, dans la mesure où de tels échanges ont souvent une portée limitée et sont généralement plus cérémonieux que ceux liés aux politiques et non controversés. Néanmoins, dans certains pays, par exemple au Royaume-Uni, en Allemagne et au Canada, le BG gère spécifiquement les échanges avec le chef de l'état. La gestion des échanges entre le gouvernement et le président est une tâche importante dans les systèmes semi-présidentiels, tels que la France (mais également le Portugal, la Finlande, et probablement la Pologne). La délimitation des compétences varie considérablement entre ces systèmes, mais l'étude de ces questions dans les systèmes semi-présidentiels dépasse largement la portée du présent document.

\subsection{Coordination des priorités stratégiques horizontales spécifiques (IE, RAP)}

Presque tous les BG sont dotés de certaines fonctions connexes, dans le cadre desquelles ils exécutent des tâches qui ne sont pas fondamentales à leurs responsabilités essentielles de gérer le système d'élaboration des politiques pour le compte du Premier ministre et du Conseil des ministres. Ces fonctions impliquent souvent des activités propres à un pays particulier ou à une période de temps donnée. Dans le premier cas, citons par exemple la gestion des affaires intergouvernementales dans les fédérations telles que le Canada et l'Allemagne, ou des sujets liés aux enfants en Roumanie. Dans le second cas de figure, on assistera par exemple à l'instauration de bureaux chargés de traiter les répercussions des inondations en Pologne, de gérer la réforme constitutionnelle au Royaume-Uni, ou de gérer l'Accord-cadre dans l'ancienne République yougoslave de Macédoine.

Cependant, certaines fonctions sont plus couramment exercées au sein des BG. En général, ces activités sont exécutées par le BG parce qu'elles sont à la fois transversales et présentent une priorité stratégique élevée, et en tant que telles, sont d'une nature qui appelle essentiellement un besoin de coordination. Par exemple, dans les états fédéraux, comme le Canada, l'Allemagne et l'Autriche, la gestion et la coordination des échanges avec les gouvernements constitutifs (Länder, provinces) sont conférées au BG. Dans les PECO et les pays des Balkans occidentaux, deux domaines de politique sont parfois traités dans le BG : réforme de l'administration publique et intégration européenne. L'assignation de ces activités au BG n'est en rien la règle universelle dans la région, comme le tableau ci-après l'indique. Cette pratique est néanmoins assez courante pour justifier une certaine discussion, notamment du fait que les conseillers étrangers recommandent souvent aux gouvernements d'assigner ces fonctions au BG. 
Tableau 7. Unités chargées d'assurer la coordination UE et RAP

\begin{tabular}{|l|c|c|}
\hline \multicolumn{1}{|c|}{ Pays membres de l'OCDE } & $\begin{array}{c}\text { Unité chargée de coordonner les échanges avec } \\
\text { l'UE }\end{array}$ & $\begin{array}{c}\text { Unité chargée de coordonner la } \\
\text { réforme }\end{array}$ \\
\hline Australie & Non applicable & BG \\
\hline Autriche & CPM Ministère des Affaires Étrangères & Ministère de la fonction publique \\
\hline France & Dans un secrétariat distinct & Ministère de l'Intérieur \\
\hline Allemagne & BG & CPM \\
\hline Islande & Ministère des Affaires Étrangères & CPM/Ministère des finances \\
\hline Irlande & CPM/Affaires étrangères & Ministère de l'Intérieur \\
\hline Pays-Bas & Ministère des Affaires Étrangères & Ministère de l'administration publique \\
\hline Espagne & Ministère des Affaires Étrangères & Ministère des finances \\
\hline Suède & CPM Ministère des Affaires Étrangères &
\end{tabular}

\begin{tabular}{|c|c|c|}
\hline PECO & $\begin{array}{l}\text { Unité chargée de coordonner les échanges avec } \\
\text { l'UE }\end{array}$ & $\begin{array}{c}\text { Unité chargée de coordonner la } \\
\text { réforme } \\
\text { de l'administration }\end{array}$ \\
\hline Bulgarie & BG & BG \\
\hline Estonie & Ministère des Affaires Étrangères & Aucune unité n'existe \\
\hline République Tchèque & Ministère des Affaires Étrangères & CPM \\
\hline Hongrie & Ministère des Affaires Étrangères & BG \\
\hline Lettonie & Bureau pour l'Intégration européenne & $\begin{array}{l}\text { BG (section au sein du Département } \\
\text { de coordination des politiques) }\end{array}$ \\
\hline Lituanie & BG CPM & Ministère de l'Intérieur \\
\hline Pologne & Ministère des Affaires Étrangères & Ministère de l'Intérieur et de \\
\hline Slovaquie & BG & Aucune unité n'existe \\
\hline Slovénie & Bureau du Gouvernement aux Affaires européennes & Ministère de l'Intérieur \\
\hline
\end{tabular}

\begin{tabular}{|l|c|c|}
\hline \multicolumn{1}{|c|}{ Pays des Balkans occidentaux } & $\begin{array}{c}\text { Unité chargée de coordonner les échanges avec } \\
\text { l'UE }\end{array}$ & $\begin{array}{c}\text { Unité chargée de coordonner la } \\
\text { réforme }\end{array}$ \\
\hline Albanie & BG (Ministre d'État pour l'intégration) & BG \\
\hline Bosnie-Herzégovine (au niveau de & Direction indépendante & Ministère de la justice \\
\hline Bosnie-Herzégovine (Fédération) & Aucune & Aucune \\
\hline Croatie & Ministère de l'intégration européenne & $\begin{array}{c}\text { Groupe de travail du Gouvernement } \\
\text { (au Ministère de l'IE) }\end{array}$ \\
\hline Ancienne République yougoslave & BG & Pourrait être rattachée au BG \\
\hline Serbie et Monténégro (Fédération) & $\begin{array}{c}\text { Ministère des échanges économiques } \\
\text { internationaux }\end{array}$ & $\begin{array}{c}\text { Bureau pour l'Organisation et } \\
\text { le Statut de l'Administration }\end{array}$ \\
\hline Serbie-Monténégro / Monténégro & Ministère de l'économie et de l'intégration \\
européenne & Ministère de la justice \\
\hline Serbie-Monténégro / Kosovo & BG & Ministère des services publics \\
\hline
\end{tabular}

De manière assez intéressante, on observe que l'intégration européenne est assignée au BG dans près de la moitié des PECO et des pays des Balkans occidentaux, alors que relativement peu de pays confient la réforme de l'administration publique à leur BG. Dans les pays membres de l'OCDE, il n'est vraiment pas courant de confier la responsabilité de la réforme de l'administration publique au BG/CPM, bien que dans quelques cas ces bureaux partagent cette responsabilité avec un ministère.

L'assignation de ces deux fonctions au Bureau du Gouvernement est-elle importante ? Bien qu'assez peu d'évaluations sur la gestion de l'intégration européenne aient été conduites dans les PECO, il semble n'y avoir aucune réponse concluante à cette question. Il semble, cependant, que cette assignation soit moins importante que ne l'est la coordination. On observe fondamentalement trois modèles d'assignation — dans le BG, dans le Ministère des Affaires Étrangères, ou dans un ministère dédié — et tous ces modèles se sont révélés efficaces. Le point majeur est que l'approche adoptée mette clairement l'accent sur la coordination des activités relatives à l'intégration européenne dans toute l'administration ; sur la reconnaissance de l'organe de coordination en tant que tel et sur le non questionnement de son autorité aussi bien au niveau bureaucratique qu'administratif ; sur la corrélation de cette activité avec les unités au sein des ministères ; et sur la dotation suffisante en personnes qualifiées. 
En ce qui concerne la réforme de l'administration publique (RAP), la situation est bien moins claire. Comme précisé ci-dessus, des consultants étrangers conseillent souvent les gouvernements d'assigner la tâche de réformer l'administration publique au Bureau du gouvernement, sous l'autorité du Premier ministre. Ce conseil se fonde sur la conviction que dans la mesure où la RAP affecte tous les ministères et les instances de l'état, et est aussi urgente que difficile, sa mise en place efficace ne peut venir que du niveau hiérarchique le plus élevé. En réalité, toutefois, les recherches à ce sujet ne sont pas très significatives, et aucune preuve empirique concluante ne vient démontrer qu'une réforme de l'administration publique est plus efficace lorsqu'elle est conduite par le BG par rapport à toute autre instance.

\section{5.}

\section{Structure des Bureaux de Gouvernement}

\subsection{Le paradoxe des différences et des similitudes}

On constate souvent que les BG diffèrent davantage d'un pays à l'autre que les ministères, comme le Ministère de l'agriculture ou le Ministère de la santé. Il existe à ceci quelques bonnes raisons. Par exemple, par rapport à la structure des ministères de tutelle, celle du BG est plus directement liée à la distribution constitutionnelle des pouvoirs. La structure du BG est sujette à des changements en réponse aux circonstances politiques (un parti contre une coalition, petite ou large, présence ou absence de vice premiers ministres). Elle est également plus tributaire des besoins et de la personnalité du Premier ministre qu'un ministère ne l'est de ceux d'un ministre donné. En effet, l'étude des organigrammes des différents BG révèle un écart important dans leur structure.

Au-delà de ces différences, dans de nombreux PECO et pays des Balkans occidentaux, le BG est lui-même en transition, ce qui explique qu'aucune structure fixe et stable ne soit encore arrêtée. Dans certains cas, on s'essaie à des expérimentations utiles pour voir ce qui fonctionne le mieux dans le système précis. Dans d'autres cas, la structure du BG peut résulter d'une assistance technique et de conseils extérieurs conflictuels, ou d'autres changements administratifs, tels que le passage d'une nouvelle loi sur la fonction publique. Quelle que soit la raison, les révisions et autres évaluations conduites dans la région au cours de ces douze dernières années ont indiqué que les fonctions et les structures des BG sont toujours en pleine mutation.

Paradoxalement, ces divergences dans les structures des BG dissimulent toutefois des similitudes fondamentales. S'il n'existe pas de BG générique, il est très facile d'en esquisser un modèle général dans lequel presque chaque pays pourrait trouver une similitude significative avec la structure de son propre BG. Les recherches ont prouvé qu'en termes de fonctions générales, les BG exercent essentiellement des activités internes au travail du gouvernement et de l'administration, plutôt que de servir ou d'avoir affaire avec le public. Les BG remplissent généralement certaines voire toutes les fonctions suivantes : fonctions logistiques et techniques liées aux sessions du CdM ; planification stratégique et programmes de travail ; coordination des politiques, conseils politiques et résolution de conflits ; fonctions juridiques ; fonctions de communication ; certaines fonctions de suivi ; et leurs propres fonctions internes de gestion. Ils remplissent plus rarement des fonctions de prestation de services, des fonctions de réglementation ou des fonctions de supervision.

La majorité des Bureaux de gouvernement sont présidés par un ministre ou un Secrétaire Général (qui est nommé soit par le gouvernement, soit par le Premier ministre). Dans la majorité des cas, le Secrétaire Général (même s'il exerce en tant que fonctionnaire) est sujet à remplacement lorsqu'un nouveau Premier ministre est nommé. Parmi les PECO, en Hongrie, en Pologne, en Slovaquie, en Slovénie et en République tchèque, le chef du Bureau de gouvernement est un ministre.

De même, parmi les pays membres de l'OCDE, la plupart des chefs des BG sont des hauts fonctionnaires, à l'exception notable de l'Allemagne et de l'Espagne.

Le plus souvent, les Bureaux de gouvernement s'organisent autour d'unités combinées (départements, secteurs, bureaux, etc.), chacune étant chargée d'exécuter les activités décrites en détail dans la section 4 du présent document. Les unités communes sont les suivantes :

- $\quad$ CPM : Le cabinet fournit un soutien administratif et politique spécifique au Premier ministre ;

- Les Cabinets des vice Premiers ministres (s'ils existent) et les cabinets des autres ministres sans portefeuille ;

- L'Unité dédiée à la presse et aux communications : Dans de nombreux cas, cette unité est rattachée au $\mathrm{CPM}$, essentiellement en raison de la nature politique des communications ; 
- $\quad$ L'Unité chargée de la préparation technique des sessions du gouvernement et des réunions des comités ministériels (s'ils existent) jouant un rôle dans le système de prise de décisions ;

- L'Unité ou les unités chargées d'assurer la coordination des politiques et de conseiller le Conseil des Ministres, le Premier ministre et les présidents des comités. Dans de nombreux cas, on observe entre 3 et 5 unités de ce type, définies en fonction de thèmes sectoriels (par exemple, politique économique, politique sociale, défense, politique extérieure);

- L'Unité de planification : Dans certains cas, il n'existe aucune unité distincte de planification, auquel cas le travail de planification incombe à l'unité chargée de la coordination des politiques ;

- $\quad$ L'Unité juridique ou législative ;

- L'Unité chargée de superviser l'application des décisions gouvernementales : Lorsque cette supervision s'avère essentiellement technique, cette fonction peut être remplie par l'unité qui prépare les sessions ;

- L'Unité chargée de gérer les échanges entre le gouvernement et le Parlement et le Bureau du Président ;

- $\quad$ L'Unité chargée de l'administration interne du BG (personnel, budget, informatique, etc.).

En outre, la plupart des BG assurent certaines fonctions connexes (évoquées au point 4.8 ci-dessus). Ces fonctions sont propres à chaque système, rendant toute tentative de généralisation impossible.

\subsection{Remarque sur la structure du CPM}

Le propos du présent document n'est pas de traiter en détail les fonctions et l'organisation du CPM, qui est la pièce de l'appareil central au service spécifique du Premier ministre en tant que chef politique du gouvernement (à la différence de la présidence des sessions du CdM). Cependant, dans le cadre de l'examen de l'organisation structurelle du BG, il semble utile d'insérer quelques commentaires à ce sujet.

Un des points fondamentaux en termes de structure est la division des responsabilités entre le BG et le CPM. Comme susmentionné, le CPM fait presque toujours partie du BG dans son acceptation administrative. En termes de fonctions, on distingue deux modèles extrêmes, avec beaucoup de différences entre eux. La première de ces deux structures extrêmes est un modèle dans lequel la plupart des fonctions essentielles (juridiques, politique, etc.) font partie du BG, laissant essentiellement au CPM la fonction restreinte de servir les besoins directs du Premier ministre, lesquels consistent par exemple en un secrétariat, un chauffeur, la gestion de son agenda, voire un rédacteur de ses discours, un attaché de presse, et un ou deux conseillers politiques personnels. Dans ce modèle, toute autre assistance au Premier ministre est fournie par le BG dans le cadre de ses services au CdM. La Bulgarie, l'ancienne République yougoslave de Macédoine et le Danemark illustrent parfaitement ce modèle. Le second modèle extrême comporte deux institutions distinctes, le CPM remplissant de nombreuses fonctions identiques à celles du BG, mais d'un point de vue politique. Le Canada et la France en sont de parfaits exemples ; le Royaume-Uni semble également évoluer dans ce sens ces dernières années.

En termes d'organisation, il semble que parmi les pays membres de l'OCDE, ces deux modèles soient plus intégrés, le chef du BG étant presque toujours également responsable du CPM. Dans les PECO et les pays des Balkans occidentaux, cette tendance incite à opérer une plus grande séparation entre le BG et CPM. Lorsque le chef du BG est également à la tête du CPM, c'est généralement à des fins administratives. En d'autres termes, le budget et le personnel relèvent de la responsabilité du BG, mais sans aucune autorité quotidienne, aucune assignation de tâche et aucune supervision de la part du chef du BG sur le personnel du CPM.

Dans la pratique, cette question est moins importante qu'il n'y paraît. Même dans les pays où on observe une organisation distincte, les deux organes partagent souvent le même emplacement ainsi que les ressources d'assistance administrative. En outre, même les pays dotés d'organisations combinées opèrent habituellement une distinction fonctionnelle claire entre les deux bureaux, en leur permettant dans la pratique de fonctionner en tant qu'organes parallèles. Dans la pratique, la séparation tranchée des fonctions et l'étroite coopération opérationnelle entre les deux organes importent bien plus que leur structure formelle. Un exercice qui exige d'opérer une distinction difficile entre les fonctions politiques d'une part et les fonctions d'élaboration de politiques et administratives de l'autre.

En conséquence, les fonctions et la structure du CPM doivent être considérées dans le contexte élargi de l'appareil administratif dans son ensemble en soutien des sessions du gouvernement, du Premier ministre, des vice-premiers ministres et des ministres sans portefeuille. Il est important de s'assurer que toutes les fonctions 
nécessaires sont bien remplies. Elles peuvent être alors assignées soit au BG soit au CPM selon les besoins et les traditions de chaque pays.

Les questions de dotation en personnel sont cruciales dans l'opération de cette différenciation. Dans cette structure générale, il est indispensable d'équilibrer la gestion des politiques, les conseils en matière d'élaboration de politiques, les propositions politiques, et le soutien technique/logistique. En conséquence de quoi, les besoins en ressources humaines sont de deux natures :

- $\quad$ un élément permanent, pour assurer la stabilité de la structure et la continuité des connaissances des procédures et des politiques, de sorte que tout changement de gouvernement ne désorganise pas les activités ni ne réduise leur efficacité ; et

- des éléments temporaires, pour tenir compte de certains conseils politiques à l'appui des opinions du Premier ministre, qui peuvent changer avec chaque PM.

Dans la plupart des pays, le BG est l'organe permanent doté essentiellement en fonctionnaires, alors que le CPM est doté en délégués politiques temporaires. C'est le cas par exemple en Lettonie, en Bulgarie et au Canada. Cependant, d'autres pays, comme la France, sont également parvenus à combiner fonctionnaires permanents et délégués politiques temporaires dans le CPM, permettant ainsi d'assurer une continuité utile en cas de remaniements gouvernementaux. 
Tableau 8. Échanges entre le BG et le CPM

\begin{tabular}{|l|c|c|}
\hline Pays membres de l'OCDE & $\begin{array}{c}\text { Le BG et le CPM constituent-ils } \\
\text { une seule instance ou deux } ?\end{array}$ & $\begin{array}{c}\text { Le chef du BG } \\
\text { préside-t-il également le CPM ? }\end{array}$ \\
\hline Australie & Deux & Non précisé \\
\hline Autriche & Une & Oui \\
\hline France & Deux & $\begin{array}{c}\text { Oui, uniquement à des fins } \\
\text { administratives }\end{array}$ \\
\hline Allemagne & Une & $\begin{array}{c}\text { Oui, uniquement à des fins } \\
\text { administratives }\end{array}$ \\
\hline Islande & Une & Oui \\
\hline Irlande & Une & Oui \\
\hline Pays-Bas & Une & Oui \\
\hline Norvège & Une & Oui \\
\hline Espagne & Deux & $\begin{array}{c}\text { Oui, uniquement à des fins } \\
\text { administratives }\end{array}$ \\
\hline Suède & Une & Oui \\
\hline
\end{tabular}

\begin{tabular}{|l|c|c|}
\hline \multicolumn{1}{|c|}{ PECO } & $\begin{array}{c}\text { Le BG et le CPM constituent-ils } \\
\text { une seule instance ou deux } ?\end{array}$ & $\begin{array}{c}\text { Le chef du BG } \\
\text { préside-t-il également le CPM } \\
?\end{array}$ \\
\hline Bulgarie & Une & Non \\
\hline République Tchèque & Une & $\begin{array}{c}\text { Oui, uniquement à des fins } \\
\text { administratives }\end{array}$ \\
\hline Estonie & Une & $\begin{array}{c}\text { Oui, uniquement à des fins } \\
\text { administratives }\end{array}$ \\
\hline Hongrie & Une & Oui \\
\hline Lettonie & Une & $\begin{array}{c}\text { Oui, uniquement à des fins } \\
\text { administratives }\end{array}$ \\
\hline Lituanie & Une & $\begin{array}{c}\text { Oui, uniquement à des fins } \\
\text { administratives }\end{array}$ \\
\hline Pologne & Une & Oui \\
\hline Slovaquie & Une & $\begin{array}{c}\text { Oui, uniquement à des fins } \\
\text { administratives }\end{array}$ \\
\hline Slovénie & Une & Non \\
\hline
\end{tabular}

\begin{tabular}{|l|c|c|}
\hline \multicolumn{1}{|c|}{$\begin{array}{c}\text { Pays des Balkans } \\
\text { occidentaux }\end{array}$} & $\begin{array}{c}\text { Le BG et le CPM constituent-ils } \\
\text { une seule instance ou deux ? }\end{array}$ & $\begin{array}{c}\text { Le chef du BG } \\
\text { préside-t-il également le CPM } \\
\text { ? }\end{array}$ \\
\hline Albanie & Deux & Non \\
\hline $\begin{array}{l}\text { Bosnie-Herzégovine (au niveau } \\
\text { de l'état) }\end{array}$ & Deux & $\begin{array}{c}\text { Oui, uniquement à des fins } \\
\text { administratives }\end{array}$ \\
\hline Bosnie-Herzégovine & Une & Non \\
\hline Croatie & Deux & Non \\
\hline $\begin{array}{l}\text { Ancienne République } \\
\text { yougoslave de Macédoine }\end{array}$ & Deux & $\begin{array}{c}\text { Oui, uniquement à des fins } \\
\text { administratives }\end{array}$ \\
\hline Serbie et Monténégro & Deux & Non précisé \\
\hline $\begin{array}{l}\text { Serbie-Monténégro / } \\
\text { Monténégro }\end{array}$ & Une & $\begin{array}{c}\text { Oui, uniquement à des fins } \\
\text { administratives }\end{array}$ \\
\hline Serbie-Monténégro / Kosovo & Une & Oui \\
\hline
\end{tabular}

\section{Conclusion : Vers un renforcement du Bureau du Gouvernement}

La présente publication a abordé un grand nombre de questions liées aux objectifs, aux fonctions et aux structures des BG dans les pays membres de l'OCDE, les PECO et les pays des Balkans occidentaux. Toutes les questions n'ont certes pas été traitées équitablement, dans la mesure où un bref examen ne suffit pas à en faire le tour. Le présent document soulève beaucoup de questions à débattre, et suggère qu'il reste beaucoup à apprendre les uns des autres si et lorsque les pays ont la volonté d'améliorer le soutien fourni au Conseil des Ministres et au Premier ministre. Dans un même temps, la diversité des seuls systèmes nationaux suggère que toute copie directe d'un modèle a de grandes chances d'échouer. 
Au cours des années, Sigma a accumulé une expérience considérable dans l'évaluation des BG et dans le soutien de leur réforme. Au nombre des enseignements les plus importants figurent notamment :

L'engagement aux plus hauts niveaux de la hiérarchie : Pour être efficaces, l'impulsion et le soutien continu pour la réforme du BG doivent venir du plus haut niveau de la hiérarchie, idéalement du Premier ministre, mais au minimum du Secrétaire Général. En outre, la volonté du PM constitue le facteur clé du soutien au système réformé.

Consolider en partant de l'organisation existante : La réforme du BG doit débuter par un examen approfondi du Bureau existant, plutôt que de partir d'un modèle abstrait. Dans la mesure où le BG existant offre une base sur laquelle se fonder - nombreuses personnes et procédures compétentes — il serait inutile et totalement irréaliste de lancer une réforme du Bureau en partant d'une feuille blanche ou du modèle d'un autre pays. Il vaut mieux consacrer du temps et des efforts pour analyser la situation actuelle et élaborer des idées de réforme sur cette base.

Valeur de l'aide extérieure : L'aide extérieure s'avère souvent utile mais, à moins de relations de travail étroites entre les experts externes et la direction et le personnel du BG, cette aide extérieure perd cruellement de sa valeur après l'évaluation initiale.

Tout changement prend du temps : L'examen initial du BG n'est pas nécessairement long, et deux mois suffisent souvent à cette analyse. En revanche, le processus d'application est long et souvent difficile, puisqu'il implique des décisions de la part du gouvernement, une planification, des changements législatifs/réglementaires, des ressources, et de la formation. Ce processus peut prendre un an ou plus avant de commencer à porter ses fruits, et pas moins de deux à trois ans pour achever l'application des changements visés.

La réforme du Bureau du Gouvernement doit se faire en parallèle avec le renforcement des moyens politiques dans les ministères : Tout coordonnateur doit avoir quelque chose à coordonner. L'ampleur du travail entrepris par le BG dépend des documents qu'il reçoit initialement des ministères. Si les ministères n'ont pas les moyens d'élaborer des propositions de grande qualité, de se consulter et d'en évaluer les impacts, le BG n'est pas en mesure de garantir une prise de décision éclairée.

Une réforme réussie exige un changement dans la culture de l'organisation : Les fonctionnaires traînent derrière eux des années de lacune et d'inexpérience dans l'exercice de leur jugement. Par le passé, les fonctionnaires ont été principalement formés à appliquer les règles ou au mieux à rechercher des données et des informations objectives. Cependant, l'élaboration et la coordination des politiques sont autant un art qu'une science et exigent par dessus tout un jugement. Bien qu'il soit admis que la prise de décisions politiques relève finalement de la responsabilité du $\mathrm{CdM}$, les ministres doivent pouvoir se fonder sur les conseils et le soutien d'experts qui font plus que se référer aux décisions et aux règles juridiques antérieures. Ils doivent avoir à leur disposition une évaluation des résultats envisageables d'une politique particulière ainsi qu'une évaluation politiquement neutre de la réaction des citoyens face à cette politique.

Il est important et possible de réformer le Bureau du Gouvernement : La capacité d'élaborer et d'appliquer une politique cohérente est essentielle à l'intégration européenne. Elle est encore plus importante pour garantir le succès de l'intégration d'un membre après son adhésion. 